\title{
$\Delta M_{\mathrm{W}} \leq 10 \mathrm{MeV} / c^{2}$ at the LHC: a forlorn hope?*
}

\author{
M.W. Krasny ${ }^{1, \text { a }}$, F. Dydak ${ }^{2}$, F. Fayette ${ }^{1}$, W. Płaczek ${ }^{3}$, A. Siódmok ${ }^{1,3}$ \\ ${ }^{1}$ LPNHE, Universités Paris VI et VII and CNRS-IN2P3, Paris, France \\ ${ }^{2}$ CERN, Geneva, Switzerland \\ ${ }^{3}$ Institute of Physics, Jagiellonian University, Cracow, Poland
}

Received: 15 April 2010 / Revised: 23 July 2010 / Published online: 1 September 2010

(C) The Author(s) 2010. This article is published with open access at Springerlink.com

\begin{abstract}
At the LHC, the measurement of the $\mathrm{W}$ mass with a precision of $\mathcal{O}(10) \mathrm{MeV} / \mathrm{c}^{2}$ is both mandatory and difficult. In the analysis strategies proposed so far, shortcuts have been made that are justified for proton-antiproton collisions at the Tevatron, but not for proton-proton collisions at the LHC. The root of the problem lies in the inadequate knowledge of parton density functions of the proton. It is argued that in order to reach a $10 \mathrm{MeV} / c^{2}$ precision for the $\mathrm{W}$ mass, more precise parton density functions of the proton are needed, and an LHC-specific analysis strategy ought to be pursued. Proposals are made on both issues.
\end{abstract}

\section{Introduction}

In much the same way as precise measurements of radiative corrections served to test and establish QED, precise measurements of input parameters and their use in the calculation of radiative corrections in the Electroweak Standard Model serve as benchmarks for new theoretical concepts. Therefore, besides the direct searches for new phenomena, the precision measurement of parameters of the Electroweak Standard Model ${ }^{1}$ - e.g., the W mass—with greater precision than available from LEP and the Tevatron, is an important and indispensable part of the LHC programme.

Whilst the $\mathrm{Z}$ mass $\left(M_{\mathrm{Z}}\right)$ is well measured to $\pm 2.1 \mathrm{MeV} / \mathrm{c}^{2}$ [1], $M_{\mathrm{W}}$ is measured at the Tevatron to $\pm 31 \mathrm{MeV} / \mathrm{c}^{2}[2]^{2}$ and at LEP to $\pm 33 \mathrm{MeV} / c^{2}$ [3]. Of the three independent

\footnotetext{
${ }^{1}$ Hereafter referred to as 'electroweak parameters'.

${ }^{2}$ The ultimate $\mathrm{W}$ mass error at the Tevatron may be as low as $\pm 15 \mathrm{MeV} / \mathrm{c}^{2}$.

*Work in part supported by the cooperation programme between the French IN2P3 and Polish COPIN Laboratories No. 05-116, and by the EU Marie Curie Research Training Network grant No. MRTN-CT2006-035505.

a e-mail: krasny@1pnhep.in2p3.fr
}

input parameters of the Electroweak Standard model, $M_{\mathrm{W}}$, $M_{\mathrm{Z}}$ and the fine-structure constant, $M_{\mathrm{W}}$ is by one order of magnitude less precise than $M_{\mathrm{Z}}$ that is second-best.

Although a precision of $M_{\mathrm{W}}$ that matches the precision of $M_{\mathrm{Z}}$ is experimentally not within reach, a much better precision than available today is desirable to exploit the full potential of the relation between $M_{\mathrm{W}}$ and the Fermi coupling constant $G_{\mathrm{F}}$ that is also well measured with a relative precision of $1 \times 10^{-5}$.

The relation between $G_{\mathrm{F}}$ and the three input parameters, $M_{\mathrm{W}}, M_{\mathrm{Z}}$ and the fine-structure constant, is a cornerstone of the Electroweak Standard Model. Radiative corrections of this relation that depend inter alia on the mass of the Higgs boson, suggest a broad range for the Higgs mass that is nevertheless well within reach at the LHC. However, in case the Higgs boson will not be found, the hunt for alternative models of electroweak symmetry breaking will be on. Then the highest possible precision of $M_{\mathrm{W}}$ will be a central issue, for a better measured relation between the quantities $G_{\mathrm{F}}, M_{\mathrm{W}}$, $M_{\mathrm{Z}}$, and the fine-structure constant, will put more stringent constraints on theoretical models.

In previous analyses, it was claimed that an $M_{\mathrm{W}}$ precision of $10 \mathrm{MeV} / \mathrm{c}^{2}$ or better will be obtained at the LHC [4-7]. This paper questions such claims and argues that shortcuts have been made that are not justified, and hence the claimed measurement precision is much too optimistic. The reason is that the analysis of $p_{\mathrm{T}, 1}$ spectra from leptonic $\mathrm{W}$ and $\mathrm{Z}$ boson decays in $\mathrm{p} \overline{\mathrm{p}}$ collisions at the Tevatron-that served as template for the respective analyses at the LHC - benefits from symmetry properties that are absent in pp collisions at the LHC. A considerably better knowledge of the $u_{\mathrm{v}}-d_{\mathrm{v}}$, $s-c$, and $b$ parton density functions (PDFs) of the pro$\operatorname{ton}^{3}$ than available today is needed, together with an LHCspecific measurement and analysis programme.

\footnotetext{
${ }^{3}$ Throughout this paper, PDFs refer to the proton.
} 
Fig. 1 Simulation of the $p_{\mathrm{T}}$ spectrum of charged leptons from the decay $\mathrm{W} \rightarrow 1 v$; the full

line is generated with zero $p_{\mathrm{T}}$ of the W's, the broken line represents the effect of a non-zero $p_{\mathrm{T}}$ as predicted by PYTHIA

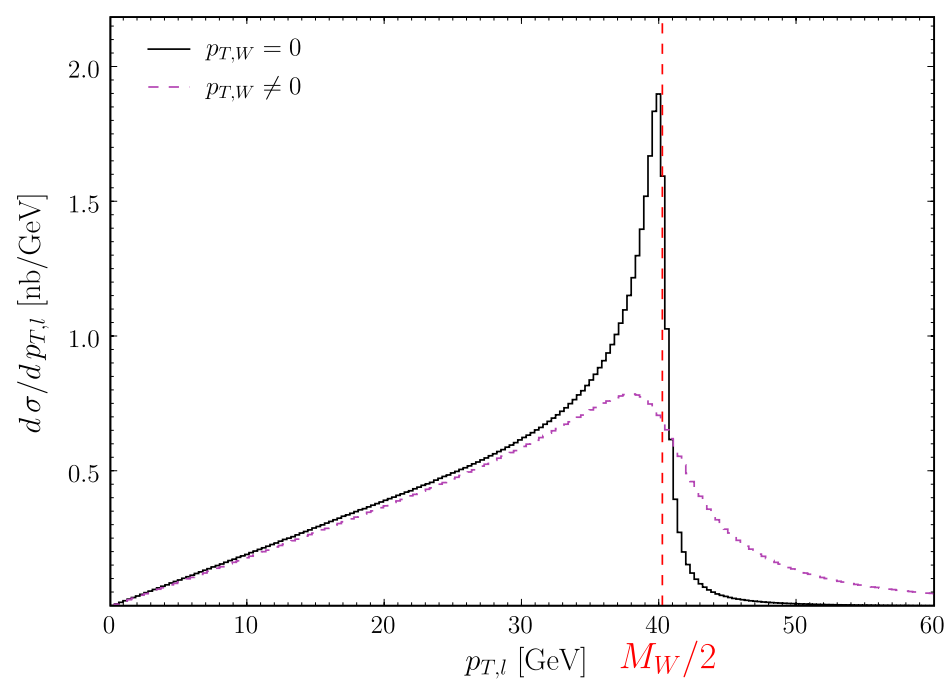

No improvement of the current situation is expected unless special experimental efforts are made to obtain the missing high-precision PDFs. Two ways forward are discussed. One is to complement the pp programme of the LHC with a deuteron-deuteron collision programme. Another is to obtain missing input from a new high-precision muon-nucleon scattering experiment, and to analyze these data coherently with LHC pp and Tevatron $\mathrm{p} \overline{\mathrm{p}}$ data.

This paper is structured as follows. Section 2 discusses the subtleties of the $\mathrm{W}$ mass measurement at the LHC, with emphasis on biases caused by the inadequate knowledge of certain PDFs.

Section 3 describes the salient features of an LHCspecific programme for the precision measurement of electroweak parameters at the LHC.

In Section 4 two experimental programmes are put forward that would permit a $10 \mathrm{MeV} / c^{2}$ precision of $M_{\mathrm{W}}$ at the LHC.

\section{Measurement of the W mass at the LHC}

Throughout this paper, it is taken for granted that the intrinsic $\mathrm{W}^{+}$and $\mathrm{W}^{-}$masses are equal. ${ }^{4}$

\subsection{The lepton transverse momentum and the scale gap}

In pp as well as in $\mathrm{p} \overline{\mathrm{p}}$ collisions, $M_{\mathrm{W}}$ is determined by the Jacobian peak in the $p_{\mathrm{T}}$ spectrum of charged leptons from $\mathrm{W} \rightarrow 1 v$ decays. The scale gap between the Jacobian peak around $40 \mathrm{GeV} / c$ and the wanted $M_{\mathrm{W}}$ precision at the $10 \mathrm{MeV} / \mathrm{c}^{2}$ level amounts to a factor of 4000 .

\footnotetext{
${ }^{4}$ The best experimental support of this assertion stems from a comparison of the measured $\mu^{+}$and $\mu^{-}$lifetimes [1], which translates into an equality of $\mathrm{W}^{+}$and $\mathrm{W}^{-}$masses at the $1.6 \mathrm{MeV} / c^{2}$ level, a precision which is out of reach at the LHC.
}

The quantitative consequences of this scale gap are highlighted in Fig. 1 which shows the change of the $p_{\mathrm{T}}$ spectrum of charged leptons from the decay $\mathrm{W} \rightarrow 1 v$ by the inclusion of what PYTHIA [8] predicts as $p_{\mathrm{T}}$ of W's at the LHC. Since the $\mathrm{W}$ mass depends on the characteristics of the Jacobian peak, it is intuitively clear that a very precise understanding of the shape of the $p_{\mathrm{T}}$ spectrum is mandatory, as is the calibration of the relation between the $p_{\mathrm{T}}$ spectrum of $\mathrm{W}$ decay leptons and $M_{\mathrm{W}}$ by means of the reference relation between the $p_{\mathrm{T}}$ spectrum of $\mathrm{Z}$ decay leptons and the precisely known $M_{Z}$. Great care must be devoted to all effects that cause either the production characteristics of $\mathrm{W}$ and $\mathrm{Z}$ to be different, or the decay characteristics of $\mathrm{W} \rightarrow 1 v$ and $\mathrm{Z} \rightarrow 1^{+} 1^{-}$ to be different, or both. Any such difference would lead to different $p_{\mathrm{T}}$ spectra of leptons from $\mathrm{W}$ decays and from the reference $Z$ decays.

The detector acceptance needs consideration, too. Since charged leptons with a pseudorapidity $|\eta|>2.5$ can hardly be measured, this limitation of the pseudorapidity range impacts on the $p_{\mathrm{T}}$ spectrum of charged leptons.

\subsection{Parton density functions}

Table 1 recalls that quite different quark-antiquark pairs contribute to the production of $\mathrm{W}^{+}, \mathrm{W}^{-}$and $\mathrm{Z}$. The following properties of the contributing quarks lead to intrinsic differences in the $p_{\mathrm{T}}$ spectra of leptons from $\mathrm{W}^{+}, \mathrm{W}^{-}$and $\mathrm{Z}$ leptonic decays: (i) their PDFs, and (ii) their weak coupling constants.

In this paper, in contrast to the usual nomenclature where a PDF is a one-dimensional function of the variable $x$, where $x$ denotes the fractional longitudinal momentum of the respective parton of the proton longitudinal momentum, what is termed 'PDF' generally refers to a two-dimensional function of $x$ and $k_{\mathrm{T}}$, where $k_{\mathrm{T}}$ is the transverse momentum of the respective parton, unless explicitly specified otherwise. 
Table 1 Quark-antiquark pairs that contribute to $\mathrm{W}^{+}, \mathrm{W}^{-}$and $\mathrm{Z}$ production

\begin{tabular}{ll}
\hline $\mathrm{W}^{+}$ & $\mathrm{u} \overline{\mathrm{d}}+\mathrm{u} \overline{\mathrm{s}}+\mathrm{ub}+\mathrm{c} \overline{\mathrm{d}}+\mathrm{c} \overline{\mathrm{s}}+\cdots$ \\
$\mathrm{W}^{-}$ & $\mathrm{d} \overline{\mathrm{u}}+\mathrm{d} \overline{\mathrm{c}}+\mathrm{s} \overline{\mathrm{u}}+\mathrm{s} \overline{\mathrm{c}}+\cdots$ \\
$\mathrm{Z}$ & $\mathrm{u} \overline{\mathrm{u}}+\mathrm{d} \overline{\mathrm{d}}+\mathrm{s} \overline{\mathrm{s}}+\mathrm{c} \overline{\mathrm{c}}+\mathrm{b} \overline{\mathrm{b}}+\cdots$ \\
\hline
\end{tabular}

The concept of a two-dimensional PDF is motivated by the large transverse momentum of the annihilating quarks in the production of $\mathrm{W}$ and $\mathrm{Z}$ which is not at the few $100 \mathrm{MeV} / \mathrm{c}$ level like for the production of low-mass particles such as pions, but rather at the level of several GeV/c. The differential of the two-dimensional PDF of the quark $\mathrm{q}, \mathrm{d} q\left(x, k_{\mathrm{T}} ; Q^{2}\right)$, denotes the number $\mathrm{d} N$ of quarks of type q with a fraction of the proton longitudinal momentum in the range $[x, x+\mathrm{d} x]$, with a transverse momentum in the range $\left[k_{\mathrm{T}}, k_{\mathrm{T}}+\mathrm{d} k_{\mathrm{T}}\right]$, at the scale $Q^{2}$. The one-dimensional PDF $q\left(x ; Q^{2}\right)$, referred to below as ' $k_{\mathrm{T}}$-integrated PDF', is the integral of the twodimensional PDF $q\left(x, k_{\mathrm{T}} ; Q^{2}\right)$ over $k_{\mathrm{T}}$. Whereas the twodimensional PDF has one longitudinal and one transverse dimension, the $k_{\mathrm{T}}$-integrated PDF has only the longitudinal dimension.

The concept of two-dimensional PDFs takes into account (i) the correlation between $x$ and $k_{\mathrm{T}}$ of the contributing quarks and antiquarks (small $x$ is correlated with large $k_{\mathrm{T}}$ ), (ii) the correlation of $k_{\mathrm{T}}$ with the hardness scale of the process (the $\mathrm{W}$ and $\mathrm{Z}$ masses are different), and (iii) the dependence of $k_{\mathrm{T}}$ on the quark type (heavier quarks have larger $k_{\mathrm{T}}$ ).

The two-dimensional PDFs are not process-independent universal functions. ${ }^{5}$ Their use is restricted to the analysis of purely leptonic observables for which the initial- and finalstate interactions can be factorized. ${ }^{6}$

Throughout this paper, the two-dimensional PDFs refer to the scale $Q^{2}=M_{\mathrm{W}}^{2}$.

Five quark flavours participate in the production of $\mathrm{W}$ and $\mathrm{Z}$. Since quarks and antiquarks are to be considered, a priori ten two-dimensional PDFs need to be known.

\section{$2.3 \mathrm{~W}$ and $\mathrm{Z}$ polarization}

By virtue of the different weak coupling constants and the different longitudinal and transverse momentum distributions of the annihilating quarks in $\mathrm{W}$ and $\mathrm{Z}$ production, the spin components in the longitudinal and transverse directions and the polarizations, respectively, are different for $\mathrm{W}^{+}, \mathrm{W}^{-}$and $\mathrm{Z}$. Because the $\mathrm{W}$ mass is determined from the $p_{\mathrm{T}}$ spectrum of decay leptons, the interest focuses on the direction perpendicular to the beam. The respective non-zero

\footnotetext{
${ }^{5}$ This is a departure from the conventional approach that considers PDFs as universal, i.e. process-independent.

${ }^{6}$ There is no gluon exchange between initial and final state.
}

spin components perpendicular to the beam direction constitute 'longitudinal' polarizations. ${ }^{7}$

The differences in the longitudinal $\mathrm{W}^{+}, \mathrm{W}^{-}$and $\mathrm{Z}$ polarizations propagate through leptonic-decay characteristics into differences of the charged-lepton $p_{\mathrm{T}}$ spectra.

\section{$2.4 \mathrm{~W}^{+}, \mathrm{W}^{-}$and $\mathrm{Z}$ leptonic-decay characteristics}

With respect to the $\mathrm{W}^{+}, \mathrm{W}^{-}$and $\mathrm{Z}$ spin directions, the angular distributions of decay leptons are different according to the $\mathrm{V}-\mathrm{A}$ and $\mathrm{V}+\mathrm{A}$ amplitudes in the boson-lepton coupling. In the $\mathrm{W}^{ \pm}$rest frame, the pure $\mathrm{V}-\mathrm{A}$ amplitude leads to the following angular distribution of the charged-lepton emission amplitude:

$w(\theta) \propto 1 \pm \cos \theta^{*}$,

where $\theta^{*}$ denotes the angle between the direction of the spin vector and charged-lepton emission. In the $\mathrm{Z}$ rest frame, the mixture of $\mathrm{V}-\mathrm{A}$ and $\mathrm{V}+\mathrm{A}$ amplitudes ${ }^{8}$ leads to the angular distribution

$w(\theta) \propto 1+\gamma \cos \theta^{*}$,

where $0<\gamma \ll 1$.

The charged-lepton emission asymmetries are modified by the Lorentz boost from the boson rest frame into the laboratory system.

On top of the genuine differences in the longitudinal polarizations of $\mathrm{W}^{+}, \mathrm{W}^{-}$and $\mathrm{Z}$ bosons, an important contribution to the differences in the $p_{\mathrm{T}}$ distribution of charged leptons in the laboratory system stems from the interference between transverse and longitudinal boson polarization amplitudes.

Altogether, from the different longitudinal polarizations of $\mathrm{W}^{+}, \mathrm{W}^{-}$and $\mathrm{Z}$, in conjunction with their different angular distributions of charged-lepton emission, and in conjunction with their momentum spectra, the question arises whether the differences of the $p_{\mathrm{T}}$ spectra of decay leptons from $\mathrm{W}^{+}, \mathrm{W}^{-}$and $\mathrm{Z}$ can be sufficiently well understood to overcome the scale gap.

\subsection{Shortcuts revisited}

There are important differences of $\mathrm{W}^{+}, \mathrm{W}^{-}$and $\mathrm{Z}$ production in pp collisions at the LHC and in $\mathrm{p} \overline{\mathrm{p}}$ collisions at the Tevatron.

In $\mathrm{p} \overline{\mathrm{p}}$ collisions at the Tevatron, there is a small forwardbackward asymmetry in the production of charged leptons from $\mathrm{Z}$ decay, and a strong asymmetry from the

\footnotetext{
${ }^{7}$ In analogy to the longitudinal polarization vector of a virtual photon.

${ }^{8}$ Because of Nature's choice of $\sin ^{2} \theta_{\mathrm{w}}$ close to $1 / 4$, the $\mathrm{V}-\mathrm{A}$ and $\mathrm{V}+\mathrm{A}$ amplitudes are nearly equal and the $\mathrm{Z}$ decay is nearly parityconserving, in contrast to $\mathrm{W}$ decay which violates parity maximally.
} 
decays of $\mathrm{W}^{+}$and $\mathrm{W}^{-}$, since e.g. $\mathrm{W}^{+}$are produced preferentially along the incoming proton direction. However, the rates and the momentum spectra of positive leptons from $\mathrm{W}^{+}$at the polar angle $\theta$ are the same as the rates of negative leptons from $\mathrm{W}^{-}$at the polar angle $\pi-\theta$. The same holds when integrated over the same range of $\theta$ and $\pi-\theta$, respectively. This lends itself to a common analysis of leptons with positive and negative charge.

In pp collisions at the LHC, there is for any of the three bosons forward-backward symmetry in the production of charged leptons: at the polar angles $\theta$ and $\pi-\theta$, the rates and the momentum spectra are identical. However, the rates and the momentum spectra are mutually different between $\mathrm{W}^{+}, \mathrm{W}^{-}$and $\mathrm{Z}$. In particular, the difference in the rates and the momentum spectra of charged leptons from $\mathrm{W}^{+}$and $\mathrm{W}^{-}$decays renders a common analysis of leptons with positive and negative charge questionable.

(a), $p \bar{p}$

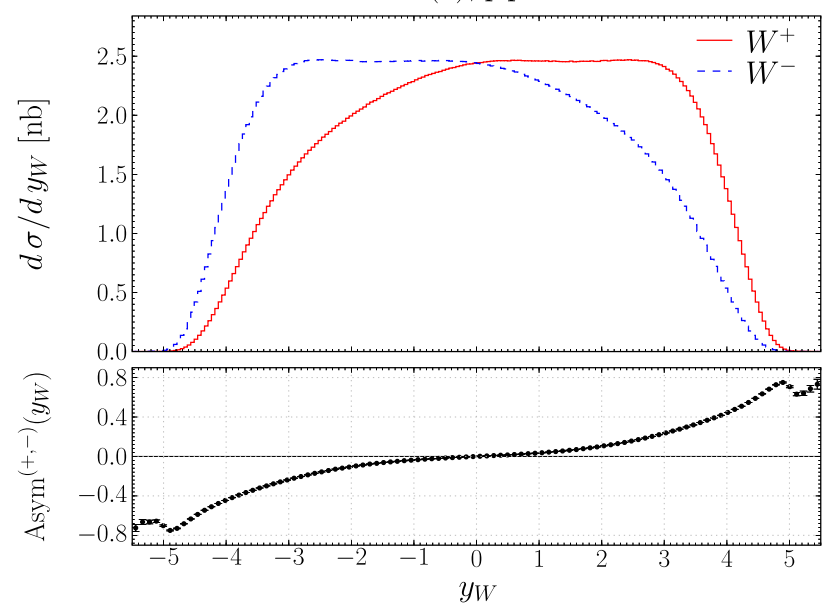

(c), $p \bar{p}$

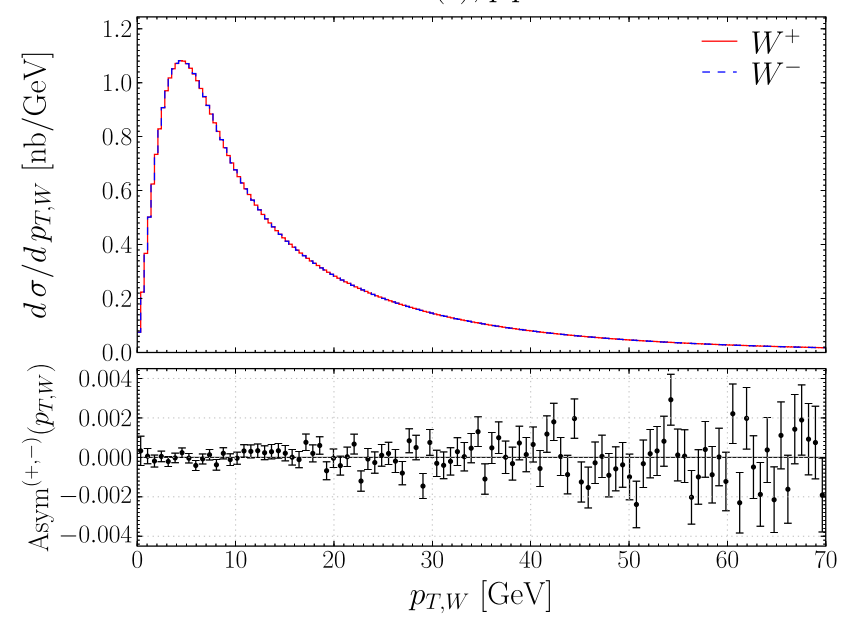

Figure 2, taken from Ref. [9], illustrates the rapidity $y$ and the $p_{\mathrm{T}}$ of $\mathrm{W}^{ \pm}$production, and Fig. 3, also taken from Ref. [9], shows the pseudorapidity and the $p_{\mathrm{T}}$ for the respective decay leptons. The difference between the characteristics of $\mathrm{W}$ production and decay in $\mathrm{p} \overline{\mathrm{p}}$ collisions and in $\mathrm{pp}$ collisions is rather striking. In pp collisions, the difference between $\mathrm{W}^{+}$and $\mathrm{W}^{-}$production is smallest at $|y| \sim 0$, because in this region the contribution from the annihilation of sea quarks with sea quarks is largest.

A common analysis of charged leptons from $\mathrm{W}^{+}$and $\mathrm{W}^{-}$ is equivalent to a $\mathrm{W}$ decay with equal $\mathrm{V}-\mathrm{A}$ and $\mathrm{V}+\mathrm{A}$ amplitudes, which is parity-conserving and resembles closely the nearly parity-conserving $\mathrm{Z}$ decay. Therefore, at the Tevatron it is straightforward and justified to calibrate in a "chargeblind' analysis the $p_{\mathrm{T}}$ spectrum of charged leptons from $\mathrm{W}$ decay with the $p_{\mathrm{T}}$ spectrum of charged leptons from $\mathrm{Z}$ decay. The ensuing systematic error of the $\mathrm{W}$ mass at the Tevatron is not dominant and comparable with the statistical error.
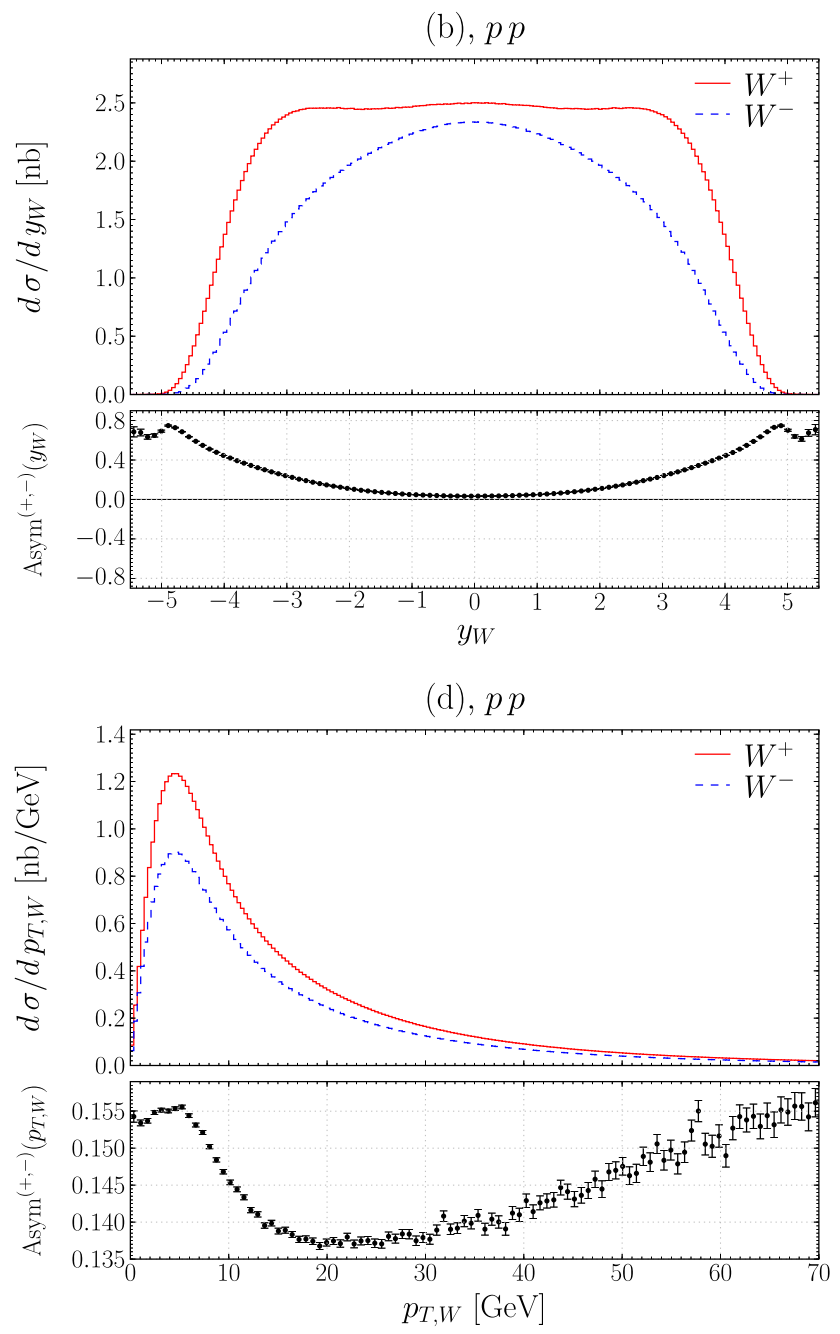

Fig. 2 Rapidity $y$ and the transverse momentum $p_{\mathrm{T}}$ of $\mathrm{W}^{ \pm}$'s, in $\mathrm{p} \overline{\mathrm{p}}$ collisions (left panels) and in pp collisions (right panels) 
(a), $p \bar{p}$

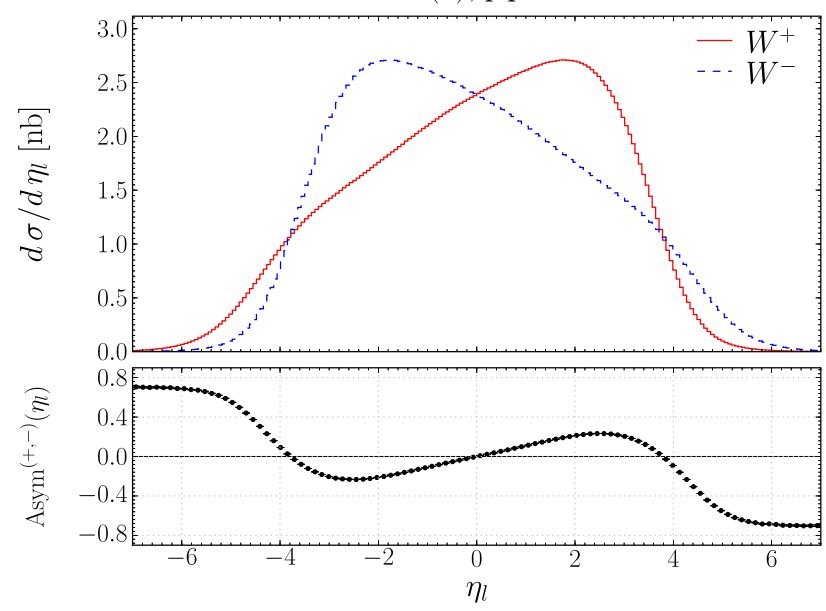

(c), $p \bar{p}$

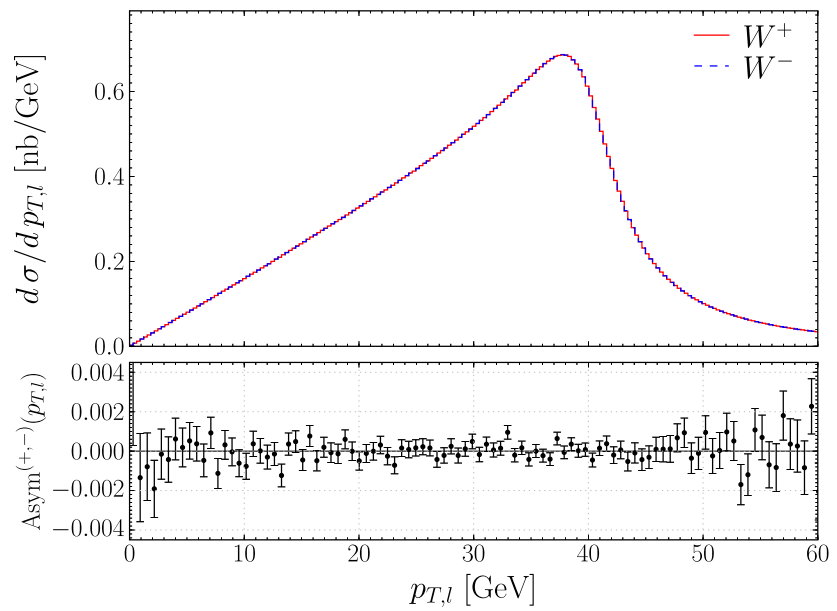

(b), $p p$

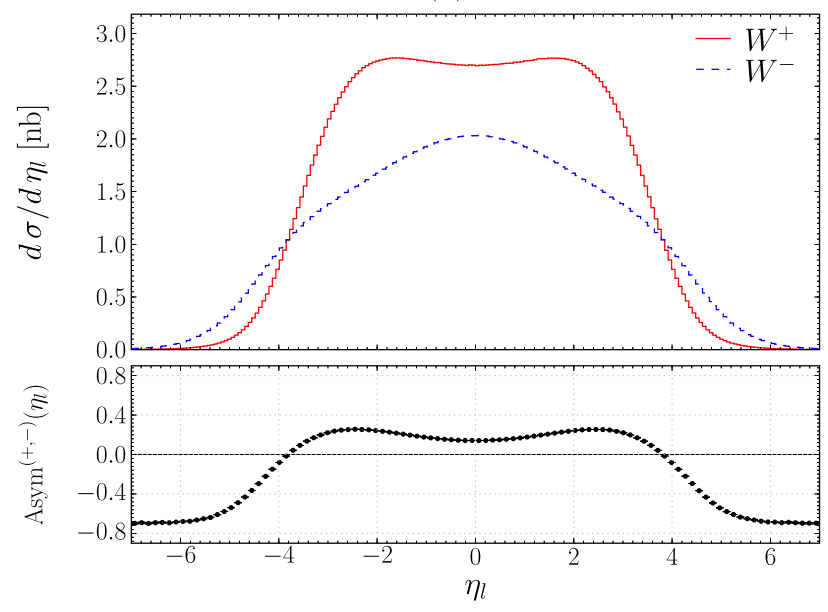

(d), $p p$

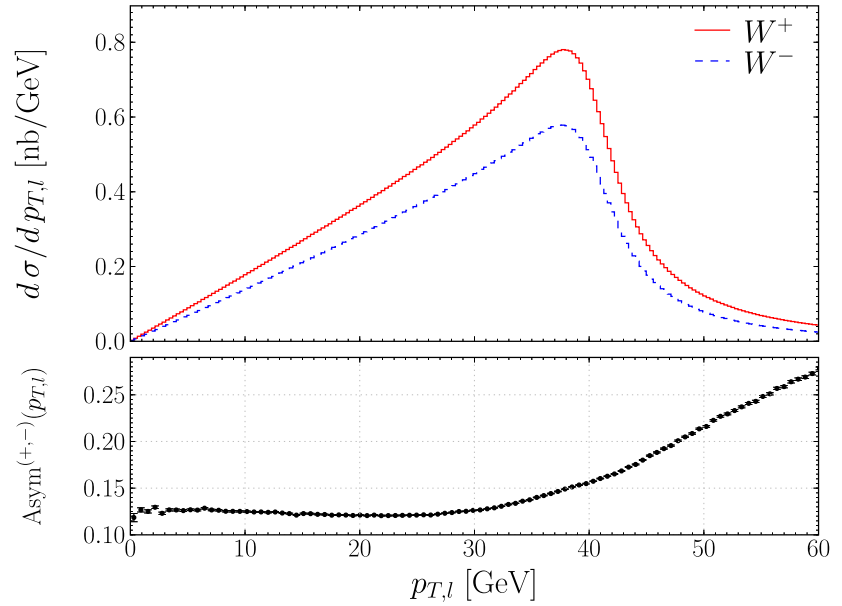

Fig. 3 Pseudorapidity $\eta$ and the transverse momentum $p_{\mathrm{T}}$ of charged leptons from the decay of $\mathrm{W}^{ \pm}$'s, in $\mathrm{p} \overline{\mathrm{p}}$ collisions (left panels) and in $\mathrm{pp}$ collisions (right panels)

At the $\mathrm{LHC}$, for the preponderance of $\mathrm{W}^{+}$over $\mathrm{W}^{-}$production in pp collisions, there is no cancellation at work that lends itself to a charge-blind analysis. If there were only sea quarks involved in the production of W's in pp collisions, symmetry between $\mathrm{W}^{+}$and $\mathrm{W}^{-}$production would not be broken. In practice, symmetry is broken by valence quarks, more specifically by the difference of the $u_{v}$ and $d_{v}$ PDFs of the proton. The charge-blind analysis that is valid for $p \bar{p}$ collisions at the Tevatron, cannot be used as template for the analysis of pp collisions at the LHC.

The effect of the valence quarks on the $p_{\mathrm{T}, 1}$ spectra of $\mathrm{W}^{+}$and $\mathrm{W}^{-}$is shown in Fig. 4. It follows that this difference, as well as the amount of sea quarks with $u$ and $d$ flavour, must be known with better precision than needed at the Tevatron. This is the LHC-specific effect from the 1 st quark family.

In Fig. 5, taken from Refs. [10, 11], the contributions of different quark-antiquark annihilations to the $\mathrm{W}^{+}, \mathrm{W}^{-}$and
$Z$ cross-sections are shown as a function of beam energy, and specifically for the Tevatron and LHC energies. The much stronger contributions from $\mathrm{c}$ and $\mathrm{b}$ quarks at the LHC energy are noteworthy.

The effects of $\mathrm{s}$ and $\mathrm{c}$ quarks on the $p_{\mathrm{T}, 1}$ spectra of $\mathrm{W}^{+}$ (left panel) and $\mathrm{W}^{-}$(right panel) in pp collisions at the LHC are shown in Fig. 6. The partner of a c quark to form a W boson, the s quark, has different distributions in $x$ and $k_{\mathrm{T}}$ than a $\mathrm{c}$ quark that is needed as partner to form a $\mathrm{Z}$ boson. Therefore, to know the difference between the s and c PDFs of the proton is important. This is the LHC-specific effect of the 2nd quark family.

Although both for $\mathrm{W}$ and $\mathrm{Z}$ production the contribution from $b$ quarks is negligible at the Tevatron, and also for $\mathrm{W}$ production at the $\mathrm{LHC}$, the contribution of $\mathrm{b}$ quarks to $\mathrm{Z}$ production is important at the $\mathrm{LHC}$ and a sufficiently precise knowledge of the PDF of the $b$ quark is needed. 
(a), $p p, W^{+}$production

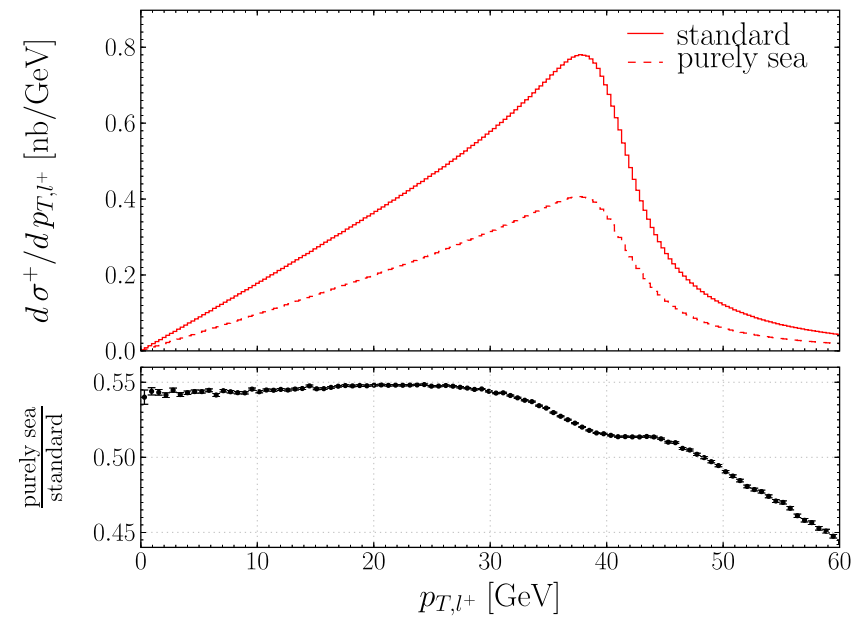

(b), $p p, W^{-}$production

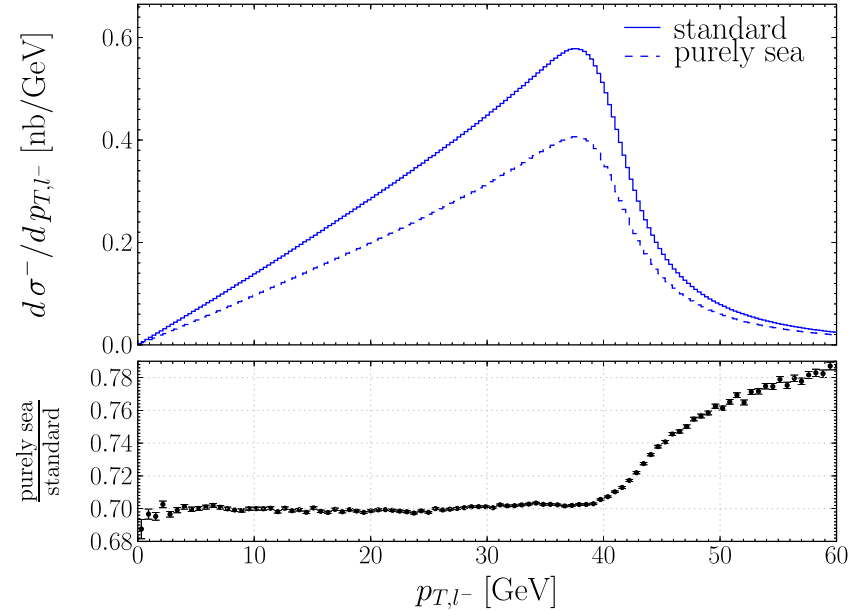

Fig. 4 The effect of the valence quarks on the $p_{\mathrm{T}, 1}$ spectra of $\mathrm{W}^{+}$(left panel) and $\mathrm{W}^{-}$(right panel) in pp collisions at the $\mathrm{LHC}$

flavour decomposition of $W$ cross sections

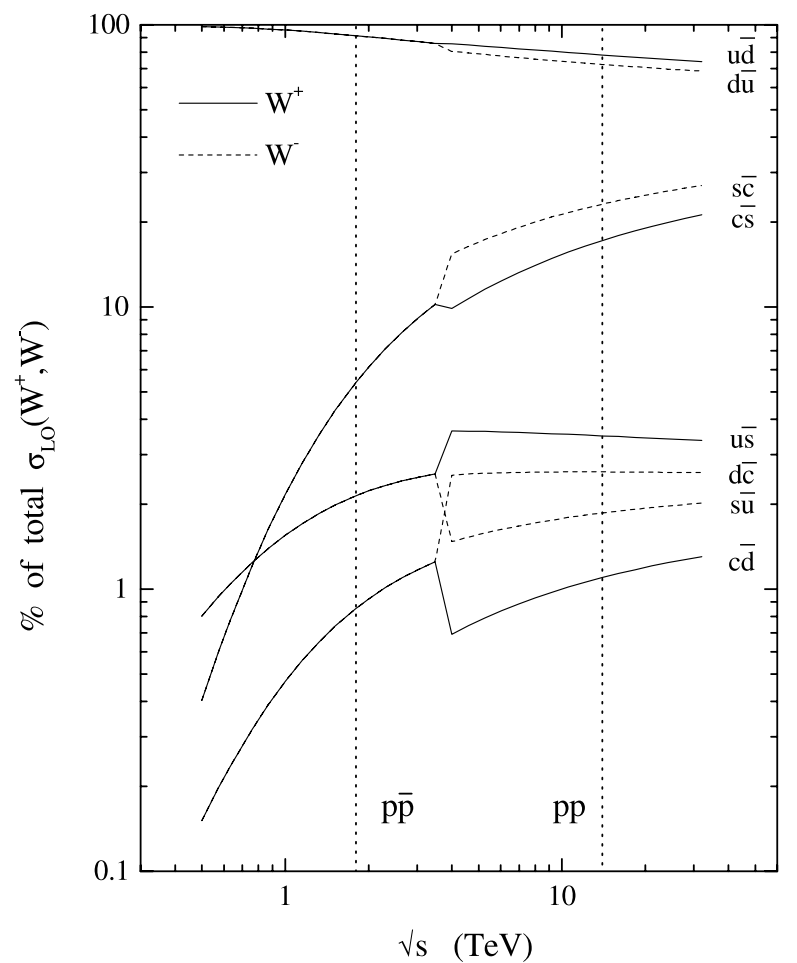

flavour decomposition of $\mathbf{Z}$ cross sections

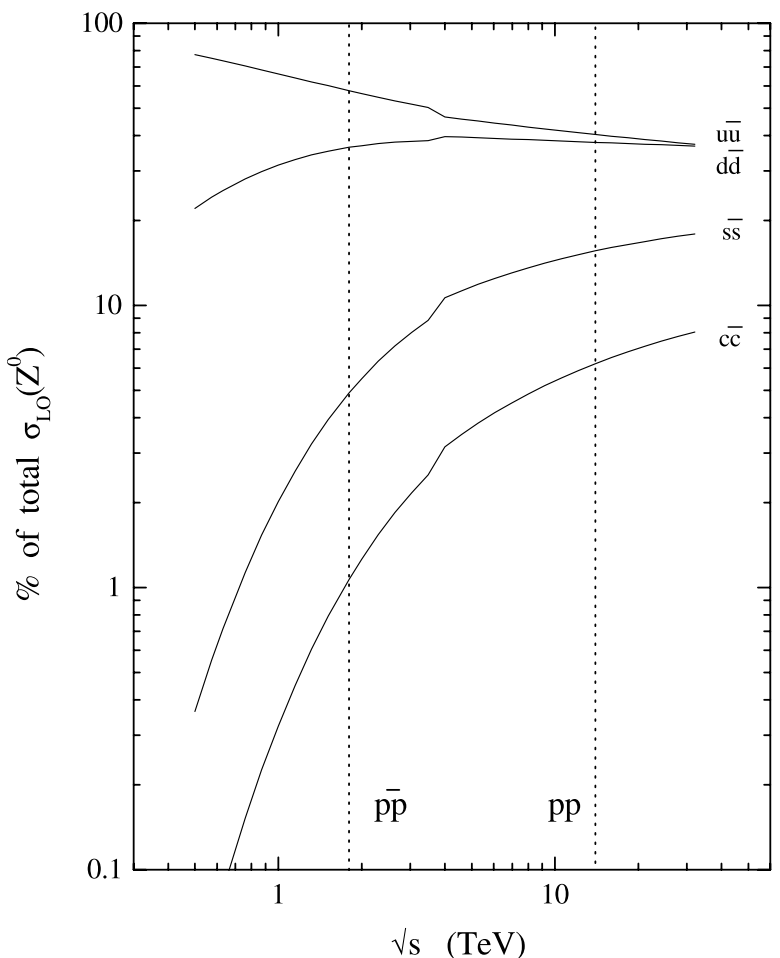

Fig. 5 Contributions of different quark-antiquark annihilations to $\mathrm{W}^{ \pm}$(left panel) and $\mathrm{Z}$ (right panel) production, as a function of the beam energy

This is the LHC-specific effect of the 3rd quark family.

The importance of the LHC-specific intricacies of the production and decay mechanisms, and their effect on the $p_{\mathrm{T}}$ spectra of decay leptons of $\mathrm{W}^{+}, \mathrm{W}^{-}$and $\mathrm{Z}$, has been missed in the LHC physics studies made so far. Not a single study made a difference between charged leptons from $\mathrm{W}^{+}$ and $\mathrm{W}^{-}$decays. As a consequence of these shortcuts, unre- alistically small errors at or below the $10 \mathrm{MeV} / \mathrm{c}^{2}$ level were reported for the $\mathrm{W}$ mass measurement at the LHC. $^{9}$

\footnotetext{
${ }^{9}$ The discussion in this paper applies mutatis mutandis also to the determination of the $\mathrm{W}$ mass from $m_{\mathrm{T}}$ spectra. The determination of $m_{\mathrm{T}}$ involves the reconstruction of the neutrino transverse momentum as missing transverse momentum. The systematic error of this measurement which involves the reconstruction of the hadronic system, is too
} 
(a), $p p, W^{+}$production

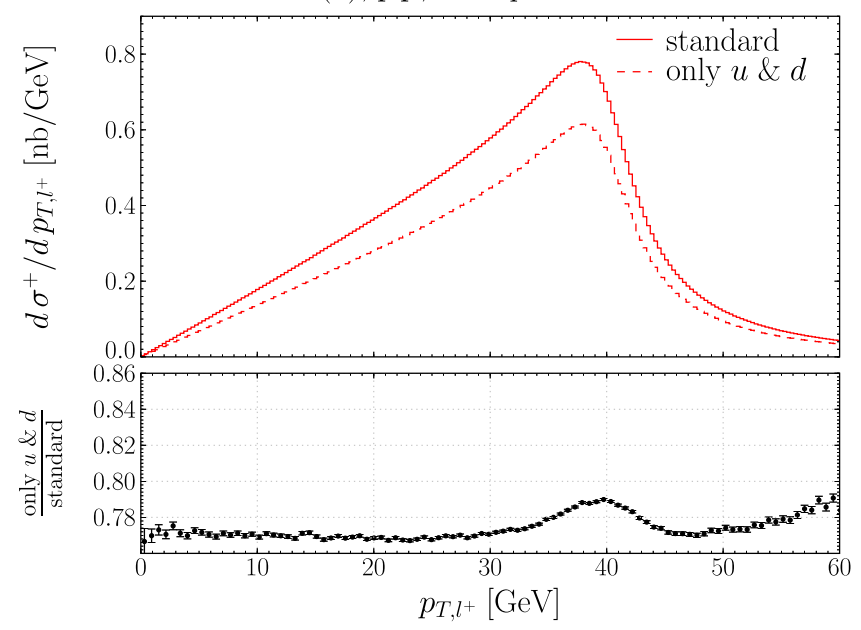

(b), $p p, W^{-}$production

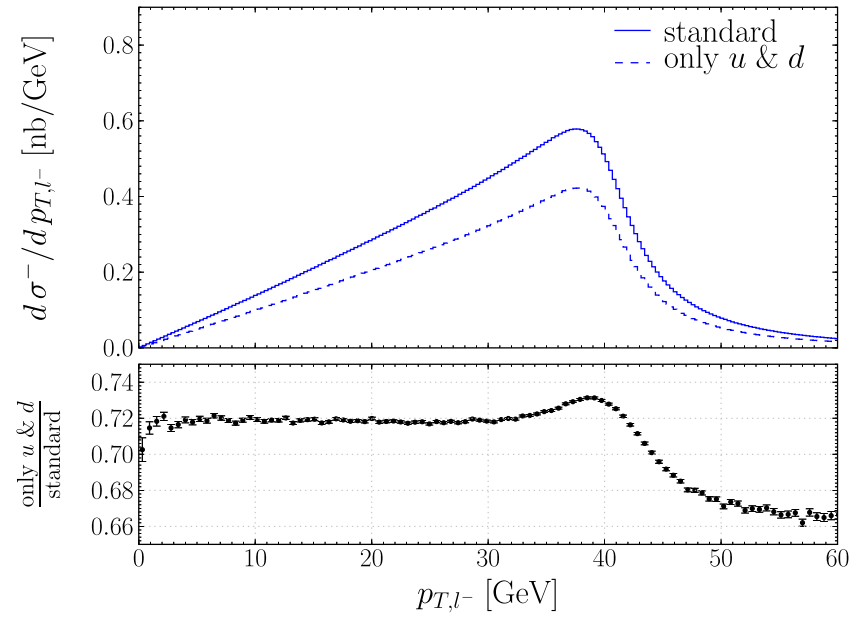

Fig. 6 The effects of $\mathrm{s}$ and $\mathrm{c}$ quarks on the $p_{\mathrm{T}, 1}$ spectra of $\mathrm{W}^{+}$(left panel) and $\mathrm{W}^{-}$(right panel) in pp collisions at the LHC

\subsection{A biased W mass}

In this section, it is argued that the current precision of pertinent proton PDFs is overestimated. It is shown that with realistic errors of these PDFs, and with correlations taken into account, the advocated $\mathrm{W}$ mass precision of $10 \mathrm{MeV} / \mathrm{c}^{2}$ or better [4-7] is much too optimistic. If the current underestimation of systematic uncertainties is not rectified the $\mathrm{W}$ mass from the LHC may be seriously biased.

The current understanding of the parton density functions may be summarized in Fig. 7 which shows the MSTW-2008 set $[10,11]$. It is advocated and widely believed that the proton PDFs are precise enough not to pose a limitation for LHC data analysis. For example, the $u_{\mathrm{v}}$ and $d_{\mathrm{v}}$ PDFs are claimed to be precise to $2 \%[10,11]$.

Why then differ the CTEQ [12] and MSTW [13] proton PDFs by much more than 2\%, as shown in Fig. 8 taken from Ref. [13], although they stem largely from the same input data?

One root of this accuracy problem is too rigid a restriction of the functional forms of the $x$ dependence of the nucleon PDFs. With a view to overcoming this problem, the NNPDF group [14] ${ }^{10}$ pioneered a Monte Carlo approach with neural networks to achieve an unbiased $x$ dependence of PDFs. However, the important progress in PDF fitting techniques cannot overcome the inherent limitations from the precision of the experimental data that determine the flavour- and valence/sea-structure of the nucleon PDFs.

The second root of the accuracy problem lies in the limitation of the CTEQ, MSTW, and NNPDF approaches to

large to be useful for the measurement of the $\mathrm{W}$ mass at the $10 \mathrm{MeV} / \mathrm{c}^{2}$ level, inter alia for reasons of reconstruction efficiency and acceptance close to the beam pipe.

${ }^{10}$ See in particular [15].

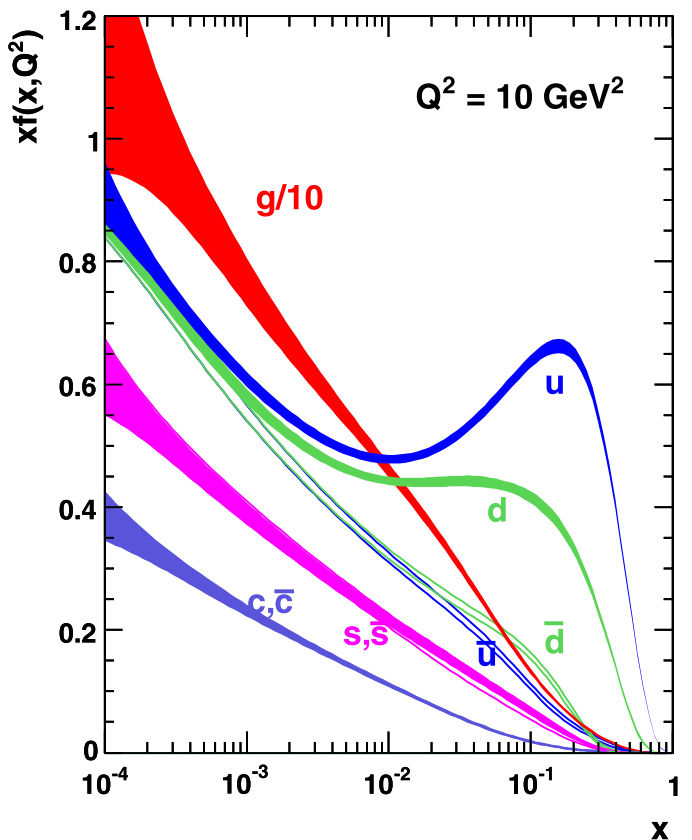

Fig. 7 The MSTW-2008 proton PDFs at $Q^{2}=10 \mathrm{GeV}^{2} / \mathrm{c}^{2}$; the widths of the bands characterize the estimated uncertainty

PDFs that depend on the longitudinal coordinate $x$ only. As discussed in Sect. 2.2, this concept neglects the correlation between $x$ and $k_{\mathrm{T}}$ of the contributing quarks and antiquarks, the correlation of $k_{\mathrm{T}}$ with the hardness scale of $\mathrm{W}$ and $\mathrm{Z}$ production, and the dependence of $k_{\mathrm{T}}$ on the quark type.

With a view to overcoming these limitations, the necessity of additional precise input to the determination of PDFs is stressed in this paper, in conjunction with the concept of two-dimensional PDFs.

In the following, a 5\% error of the $x$ dependence of the PDFs of the $u_{\mathrm{v}}$ and $d_{\mathrm{v}}$ is considered as a realistic estimate. 

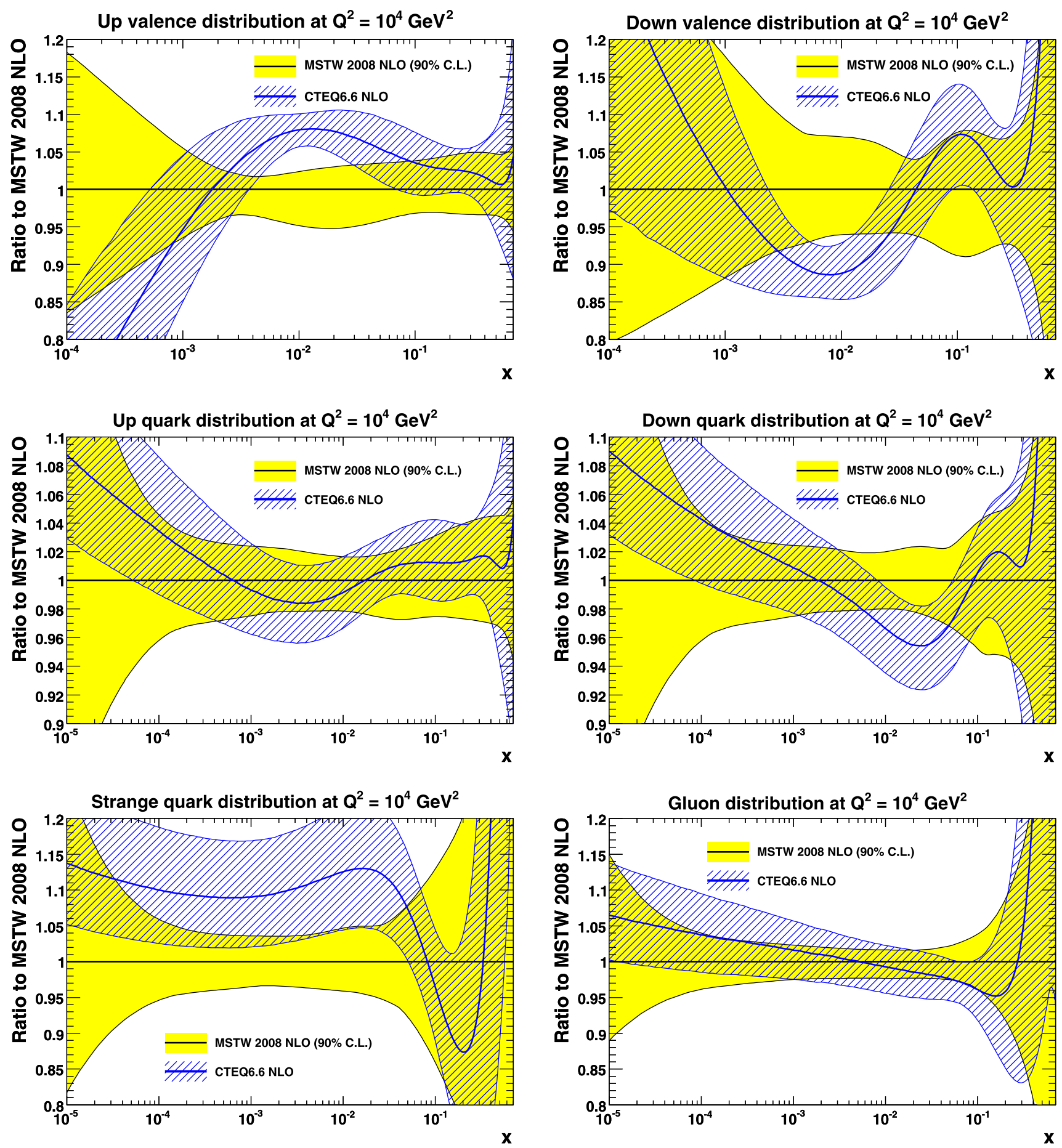

Fig. 8 Comparison of the CTEQ6.6 and MSTW2008 (NLO) PDFs of $u_{v}, d_{v}, u, d$ and s quarks, and of gluons

The present experimental uncertainty of the PDF of the $\mathrm{c}$ quark is at the $10 \%$ level, ${ }^{11}$ see Fig. 9 taken from Ref. [13]. The present experimental uncertainty of the PDF

\footnotetext{
${ }^{11}$ Theoretical calculations of heavy-quark PDFs from the gluon PDF are claimed to have a smaller error margin.
}

of the b quark is at the $20 \%$ level, see Fig. 10 taken from Ref. [13].

Another problem for the use of current proton PDFs in the analysis of $\mathrm{W}$ and $\mathrm{Z}$ production and decay at the LHC arises from 'compensating' PDF changes: a change of the PDF of one quark can be compensated by a change of the $\mathrm{PDF}$ of the other quark of the same family that leaves the $\mathrm{Z}$ 
Fig. 9 The measured PDF of the c quark, and the MSTW2008 fit, at different values of $Q^{2}$

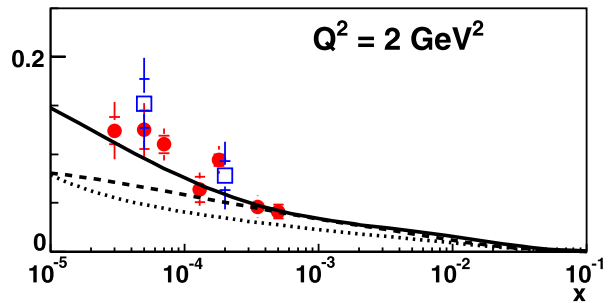

Charm structure function, $F_{2}^{c \bar{c}}\left(x, Q^{2}\right)$
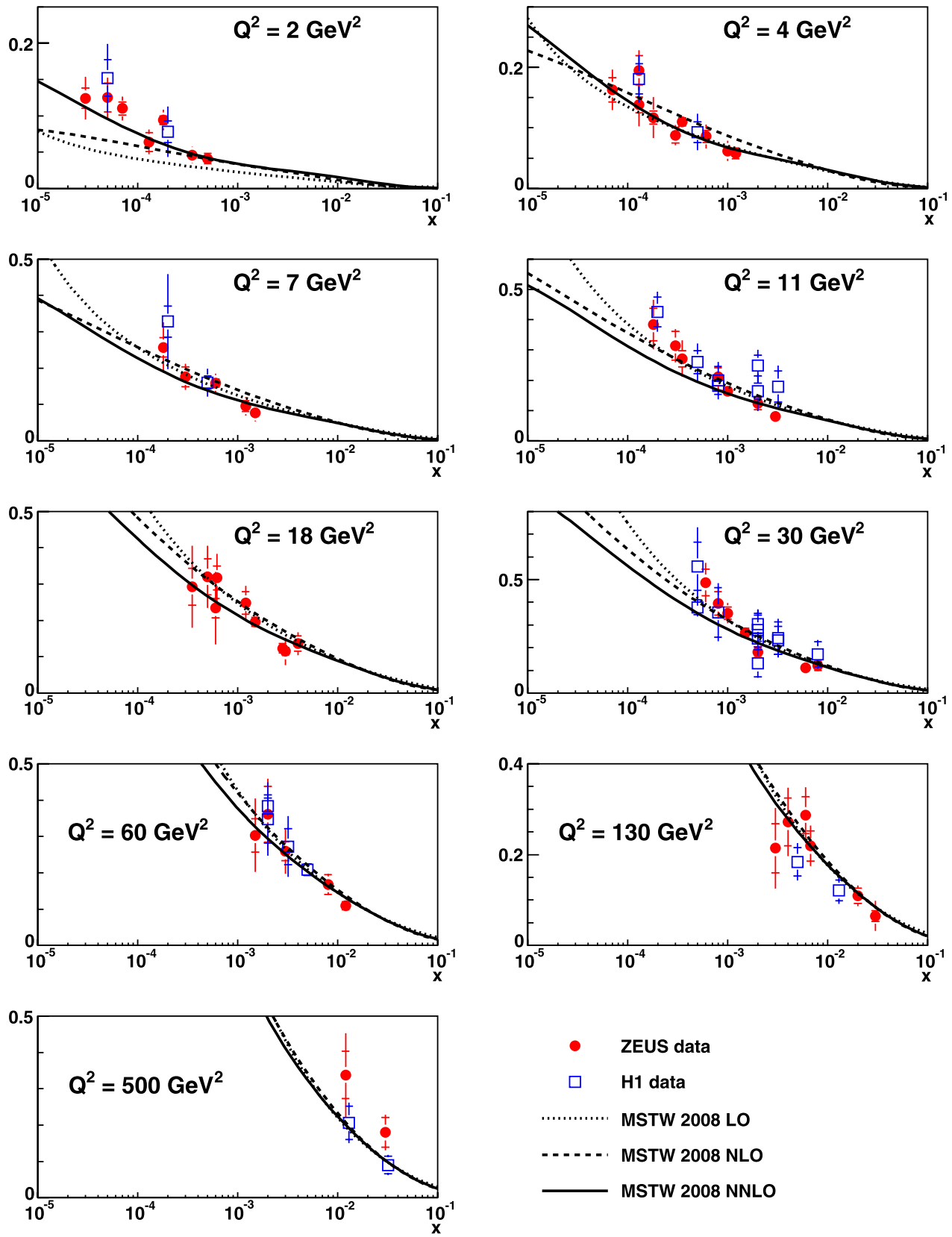

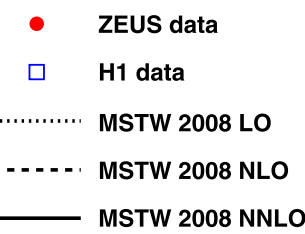

rapidity distribution nearly invariant and hence escapes detection. $^{12}$

For the 3rd quark family compensating PDF changes are obviously not possible.

\footnotetext{
${ }^{12}$ The condition of invariance of the $\mathrm{Z}$ rapidity distribution, and hence invisibility even in high-statistics data samples, is decisive: if the measured $\mathrm{Z}$ rapidity distribution looked differently than expected from the current proton PDFs, an appropriate change of the proton PDFs would be unavoidable.
}

The above uncertainties of PDFs are incorporated in the simulation of $p_{\mathrm{T}}$ spectra from $\mathrm{W}^{+}, \mathrm{W}^{-}$and $\mathrm{Z}$ leptonic decays. This simulation uses the LHAPDF package [16] of PDFs, and PYTHIA 6.4 [8] for the modelling of the QCD/QED initial-state parton shower and its hadronization; the transverse momentum $k_{\mathrm{T}}$ of quarks and antiquarks is the one incorporated in PYTHIA. The tool for event generation is WINHAC 1.31 [17], a Monte Carlo generator for single W production in hadronic collisions, and subsequent leptonic decay. WINHAC includes also neutral-current processes with $\gamma$ and $\mathrm{Z}$ bosons in the intermediate state. The novel fea- 
Fig. 10 The measured PDF of the $\mathrm{b}$ quark, and the MSTW2008 fit, at different values of $Q^{2}$
Beauty structure function, $F_{2}^{\mathrm{b} \bar{b}}\left(x, Q^{2}\right)$
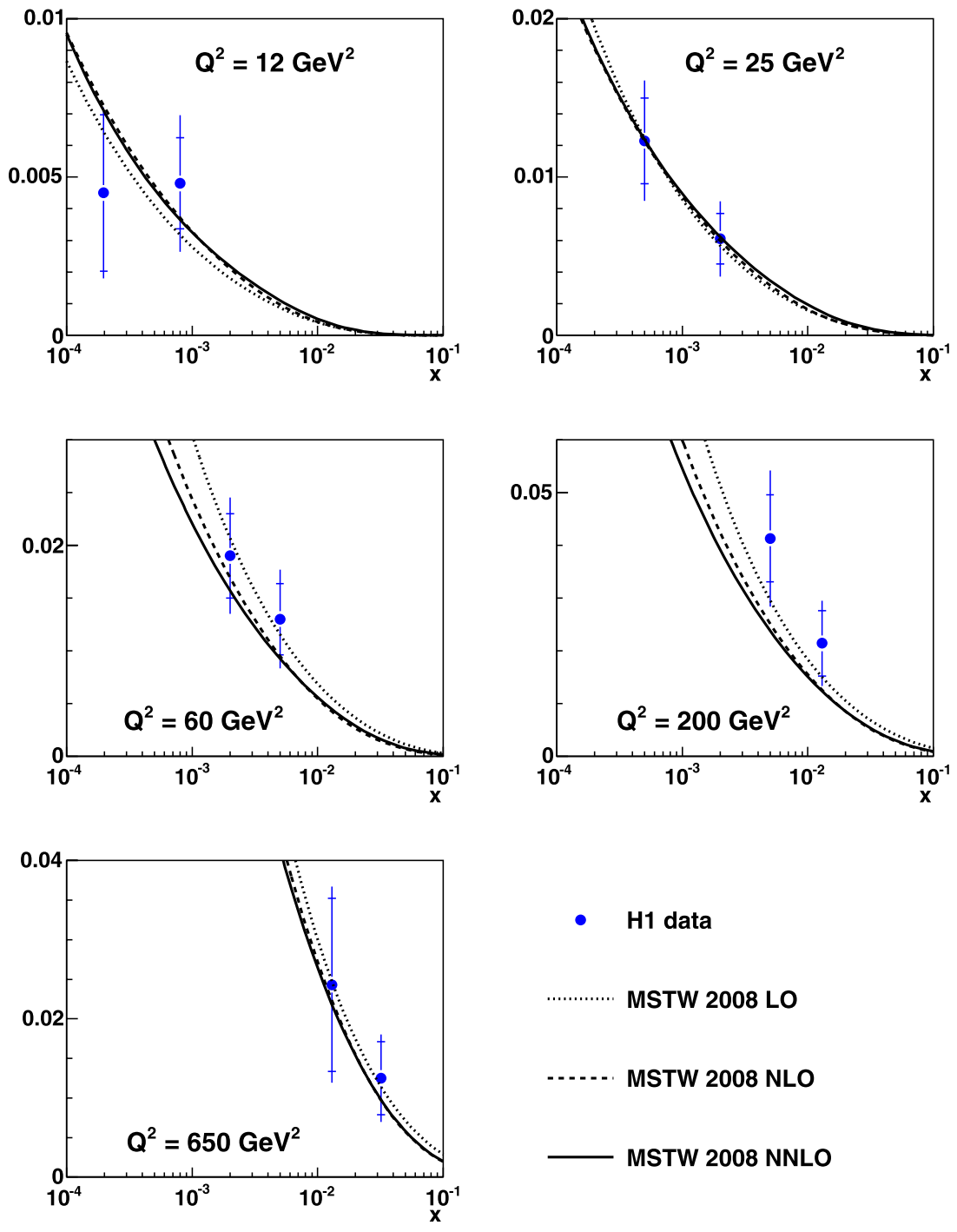

- H1 data

MSTW 2008 LO

\section{MSTW 2008 NLO}

MSTW 2008 NNLO ture of WINHAC is that it describes $\mathrm{W}$ and $\mathrm{Z}$ production and decay in terms of spin amplitudes [18]. These involve, besides all possible spin configurations of the $\mathrm{W}$ and $\mathrm{Z}$ bosons, also the ones of the initial- and final-state fermions. The advantage of this approach is that one has explicit control over all spin states, and thus over transverse and longitudinal boson polarization amplitudes and their interferences.

As an example LHC detector, ATLAS is chosen. Charged leptons from $\mathrm{W}$ and $\mathrm{Z}$ decays are accepted with $p_{\mathrm{T}}>$ $20 \mathrm{GeV} / c$ and $|\eta|<2.5$. The approximate range of $x$ for $\mathrm{W}$ and $\mathrm{Z}$ production in the above kinematical region is $5 \times 10^{-2}$ to $7 \times 10^{-4}$. The event statistics correspond to an integrated luminosity of $10 \mathrm{fb}^{-1}$. Both the electron- and muon decay channels of $\mathrm{W}$ and $\mathrm{Z}$ are considered. Since in pp collisions the spectra of positive and negative leptons are to be analyzed separately, it is natural to make the same distinction also for the leptons from $\mathrm{Z}$ decay. Along this line of rea- soning, ' $\mathrm{Z}^{+}$' and ' $\mathrm{Z}$ ', lepton $p_{\mathrm{T}}$ spectra are generated, in analogy to ' $\mathrm{W}^{+}$' and ' $\mathrm{W}^{-}$, lepton $p_{\mathrm{T}}$ spectra. ${ }^{13}$ All spectra are generated with various proton PDF configurations. The $\mathrm{Z}^{+}$and $\mathrm{Z}^{-}$lepton $p_{\mathrm{T}}$ spectra are corrected for the evolution from $Q^{2}=M_{\mathrm{W}}^{2}$ to $Q^{2}=M_{\mathrm{Z}}^{2}$.

From a fit of the Jacobian peaks in the $p_{\mathrm{T}}$ distributions and by calibrating with the known $\mathrm{Z}$ mass, the $\mathrm{W}^{+}$and $\mathrm{W}^{-}$ masses are determined.

For technical reasons, not $M_{\mathrm{W}^{+}}$and $M_{\mathrm{W}^{-}}$are separately determined but, equivalently, the average $\left(M_{\mathrm{W}^{+}}+\right.$ $\left.M_{\mathrm{W}^{-}}\right) / 2=M_{\mathrm{W}}$ and the difference $M_{\mathrm{W}^{+}}-M_{\mathrm{W}^{-}}$of the masses.

\footnotetext{
${ }^{13}$ This appears appropriate as a non-zero longitudinal $\mathrm{Z}$ polarization causes the $p_{\mathrm{T}}$ spectra of the positive and negative decay leptons to be slightly different, for the charge-dependent correlation of the $\mathrm{Z}$ spin with the emission of charged decay leptons.
} 
Table 2 lists the biases of $M_{\mathrm{W}}$ and of $M_{\mathrm{W}^{+}}-M_{\mathrm{W}^{-}}$ caused by compensating changes of the PDFs of quarks of the 1 st family. ${ }^{14}$ If the lepton rapidity range is reduced from $|\eta|<2.5$ to $|\eta|<0.3$, the quoted errors would reduce roughly by a factor of two.

Table 3 lists the biases of $M_{\mathrm{W}}$ and of $M_{\mathrm{W}^{+}}-M_{\mathrm{W}^{-}}$ caused by compensating changes of the PDFs of quarks of the 2nd family.

Table 4 lists the biases of $M_{\mathrm{W}}$ caused by changes of the PDF of the $b$ quark.

The conclusion is, when allowing for compensating PDF changes and a realistic PDF error margin, that there is no way to obtain $M_{\mathrm{W}}$ with a precision at the $10 \mathrm{MeV} / c^{2}$ level with the currently available proton PDFs.

Can the pertinent PDFs be improved with data from ongoing lepton-nucleon scattering experiments, from the Tevatron, or from the LHC? As will be discussed in Sect. 4, the answer is no. New avenues of experimentation are asked for.

\section{An LHC-specific programme}

A charge-blind analysis like for $\mathrm{p} \overline{\mathrm{p}}$ collisions at the Tevatron is not appropriate for pp collisions at the LHC. But there are more good reasons to think of a generic, LHC-specific, measurement and analysis programme:

1. The measurement of electroweak parameters should be based on a set of observables with reduced sensitivity to systematic measurement errors and to theoretical uncertainties of perturbative and non-perturbative QCD.

2. The two-dimensional PDFs in the $\mathrm{W}$ and $\mathrm{Z}$ analysis at the LHC should be defined such that compatibility is maintained with the $k_{\mathrm{T}}$-integrated PDFs used at the Tevatron, and with the missing high-precision $k_{\mathrm{T}}$-integrated PDFs that are to come from new experimental avenues.

3. To avoid theoretical uncertainties in the modelling of the lepton $p_{\mathrm{T}}$ distributions in leptonic $\mathrm{W}$ and $\mathrm{Z}$ decays, $Q^{2}$-scale effects should be determined from the data.

4. To calibrate the lepton momentum with sufficient precision, special data-taking actions should be undertaken.

An LHC-specific measurement and analysis programme along these lines is outlined below.

\subsection{Inclusive cross-sections of charged leptons from $\mathrm{W}$ and $\mathrm{Z}$ decay}

At hadron colliders, the best precision of electroweak parameters is expected from leptons from purely leptonic decays

\footnotetext{
${ }^{14}$ The differences $M_{\mathrm{W}^{+}}-M_{\mathrm{W}^{-}}$are taken from Ref. [9].
}

Table 2 Biases from uncertainties in the 1st quark family

\begin{tabular}{lll}
\hline & $\Delta M_{\mathrm{W}}$ & $\Delta\left[\left(M_{\mathrm{W}^{+}}-M_{\mathrm{W}^{-}}\right)\right]$ \\
\hline$u_{\mathrm{v}}^{\text {bias }}=1.05 u_{\mathrm{v}}$ & $+79 \mathrm{MeV} / c^{2}$ & $+115 \mathrm{MeV} / c^{2}$ \\
$d_{\mathrm{v}}^{\text {bias }}=d_{\mathrm{v}}-0.05 u_{\mathrm{v}}$ & & \\
$u_{\mathrm{v}}^{\text {bias }}=0.95 u_{\mathrm{v}}$ & $-64 \mathrm{MeV} / c^{2}$ & $-139 \mathrm{MeV} / c^{2}$ \\
$d_{\mathrm{v}}^{\text {bias }}=d_{\mathrm{v}}+0.05 u_{\mathrm{v}}$ & & \\
\hline
\end{tabular}

Table 3 Biases from uncertainties in the 2nd quark family

\begin{tabular}{lll}
\hline & $\Delta M_{\mathrm{W}}$ & $\Delta\left[\left(M_{\mathrm{W}^{+}}-M_{\mathrm{W}^{-}}\right)\right]$ \\
\hline$c^{\text {bias }}=0.9 c$ & $+148 \mathrm{MeV} / c^{2}$ & $+17 \mathrm{MeV} / c^{2}$ \\
$s^{\text {bias }}=s+0.1 c$ & & \\
$c^{\text {bias }}=1.1 c$ & $-111 \mathrm{MeV} / c^{2}$ & $-11 \mathrm{MeV} / c^{2}$ \\
$s^{\text {bias }}=s-0.1 c$ & & \\
\hline
\end{tabular}

Table 4 Biases from uncertainties in the 3rd quark family

\begin{tabular}{ll}
\hline & $\Delta M_{\mathrm{W}}$ \\
\hline$b^{\text {bias }}=1.2 b$ & $+42 \mathrm{MeV} / c^{2}$ \\
$b^{\text {bias }}=0.8 b$ & $-39 \mathrm{MeV} / c^{2}$ \\
\hline
\end{tabular}

of $\mathrm{W}$ and Z. Since the kinematical variables of neutrinos can only be inferred from measurements involving hadrons and hence are subject to larger measurement uncertainties, only observables based on charged leptons $1^{ \pm}$(more specifically: electrons and muons) are considered.

There are three classes: events with one $1^{+}$, events with one $1^{-}$, and events with one oppositely charged lepton pair $1^{+} 1^{-}$. It is assumed that these events result from the decays of $\mathrm{W}^{+}, \mathrm{W}^{-}$, and $\mathrm{Z}^{15}$ Corrections for acceptance, trigger efficiency, resolution effects, and losses from selection cuts have been applied. ${ }^{16}$ All background is assumed to be subtracted.

The following five inclusive cross-sections are measured:

$$
\begin{aligned}
& \Sigma_{\mathrm{W}^{+}}\left(p_{\mathrm{T}, 1}, \eta_{1}\right)=\mathrm{d}^{2} \sigma /\left(\mathrm{d} p_{\mathrm{T}, 1^{+}} \mathrm{d} \eta_{1^{+}}\right), \\
& \Sigma_{\mathrm{W}^{-}}\left(p_{\mathrm{T}, 1}, \eta_{1}\right)=\mathrm{d}^{2} \sigma /\left(\mathrm{d} p_{\mathrm{T}, 1^{-}} \mathrm{d} \eta_{1^{-}}\right), \\
& \Sigma_{\mathrm{Z}}\left(M_{11}, p_{\mathrm{T}, 11}, y_{11}\right)=\mathrm{d}^{3} \sigma /\left(\mathrm{d} M_{11} \mathrm{~d} p_{\mathrm{T}, 11} \mathrm{~d} y_{11}\right), \\
& \Sigma_{\mathrm{Z}^{+}}\left(M_{11}, p_{\mathrm{T}, 11}, y_{11}, p_{\mathrm{T}, 1}, \eta_{1}\right) \\
& \quad=\mathrm{d}^{5} \sigma /\left(\mathrm{d} M_{11} \mathrm{~d} p_{\mathrm{T}, 11} \mathrm{~d} y_{11} \mathrm{~d} p_{\mathrm{T}, 1^{+}} \mathrm{d} \eta_{1^{+}}\right), \\
& \Sigma_{\mathrm{Z}^{-}}\left(M_{11}, p_{\mathrm{T}, 11}, y_{11}, p_{\mathrm{T}, 1}, \eta_{1}\right)
\end{aligned}
$$

\footnotetext{
${ }^{15}$ Throughout this paper, $\mathrm{Z}$ stands for $\mathrm{Z} / \gamma$.

${ }^{16}$ Methods of selecting event samples which minimize biases in acceptance and efficiency corrections between $\mathrm{W}$ and $\mathrm{Z}$ are discussed in Ref. [19].
} 


$$
=\mathrm{d}^{5} \sigma /\left(\mathrm{d} M_{11} \mathrm{~d} p_{\mathrm{T}, 11} \mathrm{~d} y_{11} \mathrm{~d} p_{\mathrm{T}, 1^{-}} \mathrm{d} \eta_{1^{-}}\right),
$$

where $p_{\mathrm{T}, 1^{+}}$and $\eta_{1^{+}}\left(p_{\mathrm{T}, 1^{-}}\right.$and $\left.\eta_{1^{-}}\right)$denote the transverse momentum and pseudorapidity of positively (negatively) charged leptons, and $M_{11}, y_{11}$ and $p_{\mathrm{T}, 11}$ the mass, rapidity and transverse momentum of the charged lepton pair. The latter two cross-sections are not independent, only one of them, or a suitable combination, can be used.

There is one correction of the above cross-sections that still needs to be applied, though: the calibration of the positive and negative lepton momenta in terms of the functions $\epsilon_{1^{+}}\left(\rho_{1}, \eta_{1}\right)$ and $\epsilon_{1^{-}}\left(\rho_{1}, \eta_{1}\right)$ which specify the relation between the true and the reconstructed radius $\rho$ of track curvature in the magnetic field of the respective spectrometer:

$\rho_{1^{ \pm}}^{\text {rec }}=\rho_{1^{ \pm}}^{\text {true }}\left[1+\epsilon_{1^{ \pm}}\left(\rho_{1}, \eta_{1}\right)\right]$.

While a dependence of the calibration functions on the azimuthal angle can be factorized out and experimentally corrected, and therefore their possible $\phi$-dependence has been dropped, the dependence on $\rho_{1}$ and $\eta_{1}$ cannot, and must be experimentally determined from the data concurrently with the measurement of the electroweak parameters.

The above cross-sections are interpreted in terms of Standard Model parameters and two-dimensional PDFs. These are for $\mathrm{W}^{ \pm}$

$u, d, s, c, \bar{u}, \bar{d}, \bar{s}, \bar{c}, M_{\mathrm{W}}, \Gamma_{\mathrm{W}}$,

and for $\mathrm{Z}$

$u, d, s, c, b, \bar{u}, \bar{d}, \bar{s}, \bar{c}, \bar{b}, M_{\mathrm{Z}}, \Gamma_{\mathrm{Z}}, \sin ^{2} \theta_{\mathrm{W}}, \alpha$,

where $M_{\mathrm{W}}, M_{\mathrm{Z}}, \Gamma_{\mathrm{W}}$ and $\Gamma_{\mathrm{Z}}$ denote the masses and widths of $\mathrm{W}$ and $\mathrm{Z}$ (assuming $M_{\mathrm{W}^{+}}=M_{\mathrm{W}^{-}}=M_{\mathrm{W}}$ and $\Gamma_{\mathrm{W}^{+}}=$ $\left.\Gamma_{\mathrm{W}^{-}}=\Gamma_{\mathrm{W}}\right), \sin ^{2} \theta_{\mathrm{W}}$ the electroweak mixing angle, and $\alpha$ the fine-structure constant. CKM matrix elements are considered as constants and dropped for reasons of simplicity. The $u, d, s, c, b, \bar{u}, \bar{d}, \bar{s}, \bar{c}, \bar{b}$ denote two-dimensional PDFs and refer to $Q^{2}=M_{\mathrm{W}}^{2}$. Their evolution from $M_{\mathrm{W}}^{2}$ to $M_{\mathrm{Z}}^{2}$ is determined from the data as will be discussed in Sect. 3.4.

Since all QCD terms, both perturbative and nonperturbative, will be determined experimentally when relating $\mathrm{W}$ and $\mathrm{Z}$ observables, the accuracy of the leading and higherorder terms in the functional forms of the cross-sections is of secondary importance. ${ }^{17}$ The functional forms of crosssections in terms of the parameters of the Electroweak Standard Model are the ones implemented in the WINHAC and ZINHAC generators [18, 20-24].

This approach differs from the one used at the Tevatron: there, the measurement of the Standard Model parameters

${ }^{17}$ One of the motivations for this approach is the non-existence of a Monte Carlo generator that provides a full representation of the interplay between QCD and electroweak effects. relies on one-dimensional $k_{\mathrm{T}}$-integrated PDFs [25, 26], and on perturbative-QCD based algorithms for the relationship of the $p_{\mathrm{T}}$ distributions of $\mathrm{W}$ and $\mathrm{Z}[27,28]$.

\subsection{Four observables}

With a view to minimizing systematic measurement errors, the observables should have little sensitivity to detection acceptances and efficiencies. For their use in the precision measurement of several electroweak parameters, the dependence of the observables on these should be as uncorrelated as possible. Further, the observables should lend themselves to the experimental determination of perturbative and nonperturbative QCD effects, and should clearly point to missing input if needed.

The following four ratios are proposed as observables:

$\mathcal{A}_{\mathrm{W}}\left(p_{\mathrm{T}, 1}, \eta_{1}\right)=\frac{\Sigma_{\mathrm{W}^{+}}\left(p_{\mathrm{T}, 1}, \eta_{1}\right)-\Sigma_{\mathrm{W}^{-}}\left(p_{\mathrm{T}, 1}, \eta_{1}\right)}{\Sigma_{\mathrm{W}^{+}}\left(p_{\mathrm{T}, 1}, \eta_{1}\right)+\Sigma_{\mathrm{W}^{-}}\left(p_{\mathrm{T}, 1}, \eta_{1}\right)}$,

$$
\begin{aligned}
& \mathcal{A}_{\mathrm{Z}}\left(y_{\mathrm{ll}}, p_{\mathrm{T}, 11}, p_{\mathrm{T}, 1}, \eta_{1}\right) \\
& =\frac{\Sigma_{\mathrm{Z}^{+}}\left(y_{11}, p_{\mathrm{T}, 11}, p_{\mathrm{T}, 1}, \eta_{1}\right)-\Sigma_{\mathrm{Z}^{-}}\left(y_{11}, p_{\mathrm{T}, 11}, p_{\mathrm{T}, 1}, \eta_{1}\right)}{\Sigma_{\mathrm{Z}^{+}}\left(y_{11}, p_{\mathrm{T}, 11}, p_{\mathrm{T}, 1}, \eta_{1}\right)+\Sigma_{\mathrm{Z}^{-}}\left(y_{11}, p_{\mathrm{T}, 11}, p_{\mathrm{T}, 1}, \eta_{1}\right)}, \\
& \mathcal{R}_{\mathrm{WZ}}\left(p_{\mathrm{T}, 1}, \eta_{1}\right)=\frac{\Sigma_{\mathrm{W}^{+}}\left(p_{\mathrm{T}, 1}, \eta_{1}\right)+\Sigma_{\mathrm{W}^{-}}\left(p_{\mathrm{T}, 1}, \eta_{1}\right)}{\Sigma_{\mathrm{Z}^{+}}\left(p_{\mathrm{T}, 1}, \eta_{1}\right)+\Sigma_{\mathrm{Z}^{-}}\left(p_{\mathrm{T}, 1}, \eta_{1}\right)},
\end{aligned}
$$

and

$\mathcal{R}_{\mathrm{Z}}^{\text {norm }}\left(p_{\mathrm{T}, 11}, y_{11}\right)=\frac{\Sigma_{\mathrm{Z}}\left(p_{\mathrm{T}, 11}, y_{11}\right)}{\Sigma_{1^{+} 1^{-}}^{\text {nom }}}$,

where

$$
\begin{aligned}
& \Sigma_{\mathrm{Z}}\left(p_{\mathrm{T}, 11}, y_{11}\right)=\int_{M_{\mathrm{Z}}-3 \Gamma_{\mathrm{Z}}}^{M_{\mathrm{Z}}+3 \Gamma_{\mathrm{Z}}} \Sigma_{\mathrm{Z}}\left(M_{11}, p_{\mathrm{T}, 11}, y_{11}\right) \mathrm{d} M_{11}, \\
& \Sigma_{\mathrm{Z}^{+(-)}}\left(y_{11}, p_{\mathrm{T}, 11}, p_{\mathrm{T}, 1}, \eta_{1}\right) \\
& \quad=\int_{M_{\mathrm{Z}}-3 \Gamma_{\mathrm{Z}}}^{M_{\mathrm{Z}}+3 \Gamma_{\mathrm{Z}}}\left[\Sigma_{\mathrm{Z}^{+(-)}}\left(M_{11}, y_{11}, p_{\mathrm{T}, 11}, p_{\mathrm{T}, 1}, \eta_{1}\right)\right] \mathrm{d} M_{11},
\end{aligned}
$$

and

$\Sigma_{1^{+} 1^{-}}^{\text {norm }}=\iiint \Sigma_{\mathrm{Z}}\left(M_{11}, p_{\mathrm{T}, 11}, y_{11}\right) \mathrm{d} M_{11} \mathrm{~d} p_{\mathrm{T}, 11} \mathrm{~d} y_{11}$.

The latter integral is over the phase space of $1^{+} 1^{-}$pairs with a back-to-back configuration in the transverse plane, in peripheral proton-proton interactions, as developed and detailed in Ref. [29]. The measurement of electroweak parameters primarily rests on lepton pairs with their invariant mass restricted to the peak region of $M_{Z}$. Lepton pairs with invariant mass below the peak region of $M_{Z}$ will play a different rôle. They will allow to determine $Q^{2}$-scale effects 
when relating cross-sections at the $M_{\mathrm{Z}}^{2}$ scale to the ones at the $M_{\mathrm{W}}^{2}$ scale (for a detailed discussion, see Sect. 3.4).

Although each of the proposed observables depends, in general, on all electroweak parameters, the respective sensitivity is different. The $\mathcal{A}_{\mathrm{W}}$ observable is primarily sensitive to $M_{\mathrm{W}^{+}}-M_{\mathrm{W}^{-}}$and $\Gamma_{\mathrm{W}^{+}}-\Gamma_{\mathrm{W}^{-}}$. The $\mathcal{A}_{\mathrm{Z}}$ observable is merely sensitive ${ }^{18}$ to the value of $\sin ^{2} \theta_{\mathrm{W}}$. The $\mathcal{R}_{\mathrm{WZ}}$ observable is primarily sensitive to $M_{\mathrm{W}}$, but shows also a nonnegligible sensitivity to $\Gamma_{\mathrm{W}}$.

All the above observables are correlated via common PDFs and QCD algorithms. The $\mathcal{A}_{\mathrm{W}}$ observable is primarily sensitive to the difference $u-d$, both for valence and sea quarks, and (to a lesser extent) to the difference $s-c$. The $\mathcal{A}_{\mathrm{Z}}$ observable is sensitive mainly to the differences between valence and sea quarks, regardless of their flavour. The $\mathcal{R}_{\mathrm{WZ}}$ observable is sensitive to the $u-d$ and $s-c$ differences, to the differences between the density functions of valence and sea quarks, and to the density function of $b$ quarks. The $\mathcal{R}_{\mathrm{Z}}^{\text {norm }}$ observable is primarily sensitive to quark-mass effects both in the longitudinal and the transverse momentum density functions of the quarks.

\subsection{Lepton momentum calibration}

Primarily to measure the $M_{\mathrm{W}^{+}}$and $M_{\mathrm{W}^{-}}$masses with a precision of $10 \mathrm{MeV} / \mathrm{c}^{2}$, the lepton momentum calibration functions $\epsilon_{1^{+}}\left(\rho_{1}, \eta_{1}\right)$ and $\epsilon_{1^{-}}\left(\rho_{1}, \eta_{1}\right)$ (see Sect. 3.1) ought to be known with the rather demanding accuracy of $2 \times 10^{-4}$ [4-6].

The proposal presented here aims first at a reduced sensitivity of electroweak parameters on the average leptonmomentum scale, i.e. $\epsilon_{1^{+}}\left(\rho_{1}, \eta_{1}\right)+\epsilon_{1^{-}}\left(\rho_{1}, \eta_{1}\right)$. This is advantageous, for the number of $\mathrm{Z}$ events will be statistically limited when subdivided into bins of $\rho_{1}$ and $\eta_{1}$, and split into time intervals. The second aim is, for the inadequacy of a charge-blind analysis at the LHC, a calibration of the momentum-scale difference between positive and negative leptons, i.e. $\epsilon_{1^{+}}\left(\rho_{1}, \eta_{1}\right)-\epsilon_{1^{-}}\left(\rho_{1}, \eta_{1}\right)$.

As for the first aim, the $\mathcal{R}_{\mathrm{WZ}}$ observable is mostly concerned, and to a lesser extent the $\mathcal{R}_{\mathrm{Z}}^{\text {norm }}$ observable, while the $\mathcal{A}_{\mathrm{W}}$ and $\mathcal{A}_{\mathrm{Z}}$ observables only weakly depend on the average lepton-momentum scale. Therefore, the discussion focuses on the $\mathcal{R}_{\text {WZ }}$ observable.

The proposal of an LHC-specific calibration procedure [19] is the following.

1. Collect data at two centre-of-mass energies $\sqrt{s_{1}}$ and $\sqrt{s_{2}}=\left(M_{\mathrm{Z}} / M_{\mathrm{W}}\right) \times \sqrt{s_{1}}$. These two settings ascertain the same momentum fractions of the quarks that annihilate to $\mathrm{W}$ and $\mathrm{Z}$, if the $\mathrm{W}$ sample is collected at $\sqrt{s_{1}}$ and the $\mathrm{Z}$ sample at $\sqrt{s_{2}}$.

\footnotetext{
${ }^{18}$ With fixed values of $M_{\mathrm{Z}}$ and $\Gamma_{\mathrm{Z}}$, assumed throughout this paper.
}

2. Reduce the current $i$ of the spectrometer magnet when running at the lower centre-of-mass energy $\sqrt{s_{1}}$ by a factor of $M_{\mathrm{W}} / M_{\mathrm{Z}}$, with a view to equalizing the radius of curvature $\rho_{1}$ for charged leptons from $\mathrm{W}$ and $\mathrm{Z}$ decays.

3. Use a modified version of the $\mathcal{R}_{\mathrm{WZ}}$ observable defined as follows:

$$
\begin{aligned}
& \mathcal{R}_{\mathrm{WZ}}^{\bmod }\left(\rho_{1}, \eta_{1}\right) \\
& \quad=\frac{\Sigma_{\mathrm{W}^{+}}\left(\rho_{1}, \eta_{1} ; s_{1}, i\left(s_{1}\right)\right)+\Sigma_{\mathrm{W}^{-}}\left(\rho_{1}, \eta_{1} ; s_{1}, i\left(s_{1}\right)\right)}{\Sigma_{Z^{+}}\left(\rho_{1}, \eta_{1} ; s_{2}, i\left(s_{2}\right)\right)+\Sigma_{Z^{-}}\left(\rho_{1}, \eta_{1} ; s_{2}, i\left(s_{2}\right)\right)} .
\end{aligned}
$$

The integrated luminosity at the reduced centre-of-mass energy can be smaller than the one at the nominal energy by a factor of ten, with a view to achieving comparable statistics of $\mathrm{W}$ and $\mathrm{Z}$ events.

It is shown in Ref. [19] and summarized in Table 5, that with the use of the $\mathcal{R}_{\mathrm{WZ}}^{\mathrm{mod}}$ rather than the $\mathcal{R}_{\mathrm{WZ}}$ observable, the sensitivity of the $\mathrm{W}$ mass measurement on the average lepton-momentum scale is reduced by two orders of magnitude. This very significant gain results from the same topology of lepton tracks in the two settings with different centreof-mass energy.

Next, the calibration of the momentum-scale difference $\epsilon_{1^{+}}\left(\rho_{1}, \eta_{1}\right)-\epsilon_{1^{-}}\left(\rho_{1}, \eta_{1}\right)$ between positive and negative leptons is discussed. The needed accuracy is $2 \times 10^{-3}$ if the $\mathrm{W}$ mass is to measured with a precision of $10 \mathrm{MeV} / \mathrm{c}^{2}$ with the assumption $M_{\mathrm{W}^{+}}=M_{\mathrm{W}^{-}}$, and $2 \times 10^{-4}$ if the $M_{\mathrm{W}^{+}}$and $M_{\mathrm{W}^{-}}$masses are measured separately, as shown in Refs. [9, 30]. This demanding accuracy at the LHC contrasts with the Tevatron case, where the possibility of a chargeblind analysis eliminates the need of a precise calibration of the momentum-scale difference between positive and negative leptons.

The 'Double Asymmetry' method, discussed in Refs. $[9,30]$, requires two running periods with opposite polarity of the spectrometer magnet. It makes use of the following modification of the $\mathcal{A}_{\mathrm{W}}$ observable:

$\mathcal{D}_{\mathrm{W}}\left(\rho_{1}\right)=\frac{1}{2}\left[\mathcal{A}_{\mathrm{W}}^{\vec{B}=B \vec{e}_{\mathrm{z}}}\left(\rho_{1}\right)+\mathcal{A}_{\mathrm{W}}^{\vec{B}=-B \vec{e}_{\mathrm{z}}}\left(\rho_{1}\right)\right]$,

Table 5 Systematic shifts of $M_{\mathrm{W}}$ caused by lepton momentum biases as defined in (8); the statistical error of $M_{\mathrm{W}}$ is $7 \mathrm{MeV} / c^{2}$, corresponding to an integrated luminosity of $10 \mathrm{fb}^{-1}$

\begin{tabular}{llc}
\hline Lepton momentum bias & $\Delta M_{\mathrm{W}}\left[\mathrm{MeV} / c^{2}\right]$ & \\
\cline { 2 - 3 } & with $\mathcal{R}_{\mathrm{WZ}}$ & with $\mathcal{R}_{\mathrm{WZ}}^{\text {mod }}$ \\
\hline$\epsilon_{\mathrm{1}^{+}}=+\epsilon_{\mathrm{1}^{-}}=+0.005$ & +226 & +5 \\
$\epsilon_{1^{+}}=+\epsilon_{1^{-}}=-0.005$ & -223 & -2 \\
$\epsilon_{1^{+}}=-\epsilon_{\mathrm{1}^{-}}=+0.005$ & +40 & +22 \\
$\epsilon_{\mathrm{1}^{+}}=-\epsilon_{\mathrm{1}^{-}}=-0.005$ & -19 & -31 \\
\hline
\end{tabular}


where $\rho_{1}$ represents the radius of the lepton track, and $B$ the magnetic field strength.

This latter method can be used for the $\mathrm{W}$ mass measurement and provides the needed calibration precision. However, as far as only the measurement of the $\mathrm{W}$ mass under the assumption $M_{\mathrm{W}^{+}}=M_{\mathrm{W}^{-}}$is concerned, what is needed can be obtained in a simpler way without changing the magnet polarity. Use is made of $1^{+} 1^{-}$pairs with invariant mass close to the $Z$ peak. Thanks to Nature's choice of $\sin ^{2} \theta_{\mathrm{W}}$ close to $1 / 4$, the difference of the $p_{\mathrm{T}}$ distributions of positive and negative leptons is minimal, while the statistics of events is large. The comparison of the $\mathrm{Z}^{+}$and $\mathrm{Z}^{-} p_{\mathrm{T}}$ distributions delivers what is wanted. The sensitivity to the precise value of $\sin ^{2} \theta_{\mathrm{W}}$ is sufficiently weak to permit to factorize out the calibration procedure at the precision level of $2 \times 10^{-3}$.

As this precision is not sufficient for separate precision measurements of $M_{\mathrm{W}^{+}}$and $M_{\mathrm{W}^{-}}$, the 'Double Asymmetry' method is indispensable for these.

The conclusion is that the momentum scale of both positive and negative leptons can be calibrated with sufficient precision so as not to limit the precision of electroweak parameters.

\section{$3.4 Q^{2}$ evolution}

The rationale to deal with $Q^{2}$ evolution builds on the concept of rescaling the LHC energy and of the field of the spectrometer magnet in such a way that production and leptonic decays of $\mathrm{W}$ and $\mathrm{Z}$ are on the same footing: for a given $\mathrm{W}$ or $Z$ rapidity, the fractions of the proton momentum carried by annihilating quarks are the same, as is the radius of curvature of leptons from $\mathrm{W}$ and $\mathrm{Z}$ decays.

The equality of the $\rho_{1}$ and $\eta_{1}$ distributions of the leptons holds exactly, though, only for collinear massless quarks with flavour-independent PDFs. Even in such an ideal case the observables proposed in this paper are still sensitive to the $Q^{2}$-dependence of the two-dimensional PDFs of the annihilating quarks. Moreover, the $\mathcal{R}_{\mathrm{WZ}}^{\mathrm{mod}}$ observable is sensitive to the relative normalization of the $\mathrm{W}$ and $\mathrm{Z}$ samples obtained in separate settings.

The $Q^{2}$-scale dependent effects concern primarily the $\mathcal{R}_{\mathrm{WZ}}^{\mathrm{mod}}$ observable, hence the following discussion refers to this observable. The generalization to other observables is straightforward.

It is proposed to select pairs of opposite-charge leptons and calculate the ratio

$\mathcal{C}_{\mathrm{QCD}}$

$$
=\frac{\int_{M_{\mathrm{Z}}-3 \Gamma_{\mathrm{Z}}}^{M_{\mathrm{Z}}+\Gamma_{\mathrm{l}^{+} \mathrm{1}^{-}}}\left(s_{2}, i\left(s_{2}\right), M_{\mathrm{1}^{+} \mathrm{1}^{-}}\right) \mathrm{d} M_{\mathrm{1}^{+} \mathrm{1}^{-}}}{\int_{M_{\mathrm{W}}-3 \Gamma_{\mathrm{W}}}^{M_{\mathrm{W}}+3 \Gamma_{\mathrm{BW}}}\left(s_{\mathrm{1}^{+} \mathrm{1}^{-}}, M_{\mathrm{W}}, \Gamma_{\mathrm{W}}\right) w_{\mathrm{EW}} N_{\mathrm{l}^{+} \mathrm{1}^{-}}\left(s_{1}, i\left(s_{1}\right), M_{\mathrm{l}^{+} \mathrm{1}^{-}}\right) \mathrm{d} M_{\mathrm{l}^{+} \mathrm{l}^{-}}}
$$

as a function of $\rho_{1}$ and $\eta_{1}$ of a randomly chosen $1^{+}$or $1^{-}$. Each pair with an invariant mass $M_{\mathrm{Z}}-3 \Gamma_{\mathrm{Z}} \leq M_{1^{+} 1^{-}} \leq$
Table 6 Systematic shifts of $M_{\mathrm{W}}$ caused by different quark $k_{\mathrm{T}}$ 's; the statistical error of $M_{\mathrm{W}}$ is $7 \mathrm{MeV} / \mathrm{c}^{2}$, corresponding to an integrated luminosity of $10 \mathrm{fb}^{-1}$

\begin{tabular}{llll}
\hline$\sigma_{k_{\mathrm{T}}}[\mathrm{GeV} / c]$ & $\Delta M_{\mathrm{W}}\left[\mathrm{MeV} / c^{2}\right]$ & \\
\cline { 2 - 4 } & with $\mathcal{R}_{\mathrm{WZ}}$ & with $\mathcal{R}_{\mathrm{WZ}}^{\text {mod }}$ & with $\mathcal{R}_{\mathrm{WZ}}^{\mathrm{QCD}}$ \\
\hline 0 & -180 & -26 & -8 \\
3 & -68 & -7 & -3 \\
6 & +206 & +12 & +4 \\
\hline
\end{tabular}

$M_{\mathrm{Z}}+3 \Gamma_{\mathrm{Z}}$ and $M_{\mathrm{W}}-3 \Gamma_{\mathrm{W}} \leq M_{1^{+} 1^{-}} \leq M_{\mathrm{W}}+3 \Gamma_{\mathrm{W}}$, respectively, is weighted with the Breit-Wigner function ${ }^{19}$

$f_{\mathrm{BW}}\left(s_{1^{+} 1^{-}}, M_{\mathrm{W}}, \Gamma_{\mathrm{W}}\right)=\frac{1}{\pi} \frac{M_{\mathrm{W}} \Gamma_{\mathrm{W}}}{\left(s_{1^{+} 1^{-}}-M_{\mathrm{W}}^{2}\right)^{2}+M_{\mathrm{W}}^{2} \Gamma_{\mathrm{W}}^{2}}$,

where $s_{1^{+} 1^{-}}=M_{1^{+} 1^{-}}^{2}$. The factor $w_{\mathrm{EW}}$ normalizes the integral of the $M_{1^{+} 1^{-}}$spectrum between $M_{\mathrm{W}}-3 \Gamma_{\mathrm{W}}$ and $M_{\mathrm{W}}+3 \Gamma_{\mathrm{W}}$ to the cross section of a $Z$-like boson with the mass and the width of the $\mathrm{W}$-boson. As a result, the numerical value of $\mathcal{C}_{\mathrm{QCD}}$ is close to unity.

In order to eliminate the $Q^{2}$-scale dependence of the twodimensional PDFs, the $\mathcal{R}_{\mathrm{WZ}}^{\mathrm{mod}}$ observable is replaced by the observable

$\mathcal{R}_{\mathrm{WZ}}^{\mathrm{QCD}}\left(\rho_{1}, \eta_{1}\right)=\mathcal{R}_{\mathrm{WZ}}^{\bmod }\left(\rho_{1}, \eta_{1}\right) \times \mathcal{C}_{\mathrm{QCD}}\left(\rho_{1}, \eta_{1}\right)$.

The detector-level simulation and the numerical evaluation of this concept is presented in Refs. [19]. Here, the result for the most sensitive electroweak parameter, the $\mathrm{W}$ mass, is summarized in Table 6 . When varying the $k_{\mathrm{T}}$ of quarks ${ }^{20}$ over the rather conservative range $0-6 \mathrm{GeV} / c$, the W mass varies with the $\mathcal{R}_{\mathrm{WZ}}$ observable between -180 and $+206 \mathrm{MeV} / \mathrm{c}^{2}$, while there is no significant variation with the $\mathcal{R}_{\mathrm{WZ}}^{\mathrm{QCD}}$ observable.

The $\mathcal{R}_{\mathrm{WZ}}^{\mathrm{QCD}}$ observable is insensitive to the precision of the relative normalization of the two data sets taken at the energies $\sqrt{s_{1}}$ and $\sqrt{s_{2}}$.

\subsection{The missing input}

At the LHC, the number of observables, (9)-(12), is four whereas a priori the number of two-dimensional PDFs (for five quark flavours $\mathrm{u}, \mathrm{d}, \mathrm{s}, \mathrm{c}, \mathrm{b}$ ) is ten = five (quark flavours) $\times$ two (quarks and antiquarks). Both the observables and the PDFs are two-dimensional functions of one longitudinal and one transverse variable.

\footnotetext{
${ }^{19}$ This formula corresponds to the so-called fixed-width scheme, however it can also be applied to the running-width scheme in which case both $M_{\mathrm{W}}$ and $\Gamma_{\mathrm{W}}$ have to be divided by the factor $\sqrt{1+\left(\Gamma_{\mathrm{W}} / M_{\mathrm{W}}\right)^{2}}$.

${ }^{20}$ Specifically: the value of the PYTHIA smearing parameter $\sigma_{k_{\mathrm{T}}}$ of the flavour-independent partonic $k_{\mathrm{T}}$ density function.
} 
The method, discussed in Sect. 3.4, of determining experimentally the $Q^{2}$ evolution of the observables from $M_{\mathrm{W}}^{2}$ to $M_{\mathrm{Z}}^{2}$, permits to define all PDFs at the $M_{\mathrm{W}}^{2}$ scale. For simplicity, their $Q^{2}$ dependence is henceforth dropped.

Can the number of PDFs be reduced to match the number of observables?

In a first step it is discussed how to determine, at fixed $x$, the ten $k_{\mathrm{T}}(x)$ densities for the $\mathrm{u}, \mathrm{d}, \mathrm{s}, \mathrm{c}, \mathrm{b}$ quarks and antiquarks. Then, in a second step, it is discussed how to determine the $x$ dependence of the ten $k_{\mathrm{T}}$-integrated PDFs.

It can be assumed that, at fixed $x$, the $k_{\mathrm{T}}$ dependence of the two-dimensional PDFs of quarks and antiquarks is the same. This is suggested because $\mathrm{W}$ and $\mathrm{Z}$ bosons are produced predominantly by the annihilations of sea quarks, hence the equality $k_{\mathrm{T}}^{\mathrm{q}}(x)=k_{\mathrm{T}}^{\overline{\mathrm{q}}}(x)$ is a reasonable assumption for all five quark flavours. ${ }^{21}$ This reduces the number of needed $k_{\mathrm{T}}$ densities from ten to five. Assuming further that the $k_{\mathrm{T}}$ densities are the same for the $\mathrm{u}$ and d quarks, the $p_{\mathrm{T}}$ dependences of the four LHC observables permit the determination of all four remaining $k_{\mathrm{T}}$ densities, $k_{\mathrm{T}}^{\mathrm{u}, \mathrm{d}}(x), k_{\mathrm{T}}^{\mathrm{s}}(x)$, $k_{\mathrm{T}}^{\mathrm{c}}(x)$, and $k_{\mathrm{T}}^{\mathrm{b}}(x)$. In practice, better precision is obtained if in addition the further assumption $k_{\mathrm{T}}^{\mathrm{u}, \mathrm{d}}(x)=k_{\mathrm{T}}^{\mathrm{s}}(x)$ is made.

As for the $x$ dependence of the ten needed $k_{\mathrm{T}}$-integrated PDFs, the equality of the quark and antiquark densities of the $\mathrm{s}, \mathrm{c}$, and $\mathrm{b}$ flavours is assumed, ${ }^{22} s(x)=\bar{s}(x), c(x)=$ $\bar{c}(x)$ and $b(x)=\bar{b}(x)$. This reduces the number of needed $k_{\mathrm{T}}$-integrated PDFs from ten to seven. Given the $\eta$ dependences of the four LHC observables, three experimental constraints are missing, sufficiently precise input PDFs are needed from elsewhere.

The following three $k_{\mathrm{T}}$-integrated flavour-singlet PDFs are least constrained by the LHC data alone: $u_{\mathrm{v}}(x)-d_{\mathrm{v}}(x)$, $s(x)-c(x)$ and $b(x)$. They are referred to below as 'missing input'. This choice of missing input is also made in other papers on this subject (Refs. [9, 19, 31, 32]).

The rationale behind the choice of the bulk of the missing input in terms of differences of PDFs is to focus attention on the possibility of compensating PDF changes that was discussed in Sect. 2.6.

The reason for the preference of the flavour-nonsinglet PDF $u_{\mathrm{v}}-d_{\mathrm{v}}$ is that it will have to be obtained from data taken at smaller $Q^{2}$ scales and subsequently extrapolated to the $M_{\mathrm{W}}^{2}$ scale. The $Q^{2}$ evolution of non-singlet PDFs is independent of the initial form of the gluon density function, hence the extrapolation uncertainty is reduced.

The crucial point is whether the missing input, taken from existing data, is precise enough.

\footnotetext{
${ }^{21}$ The contributions from valence quarks calls for a small correction to be applied.

${ }^{22}$ For s quarks, a small violation of this equality is likely which calls for a small correction to be applied; however, for $\mathrm{u}$ and $\mathrm{d}$ quarks, at $x \sim 6 \times 10^{-3}$ such equality is violated at the level of $\sim 15 \%$.
}

The uncertainties in the missing input used in the pertinent studies in Refs. [9, 19, 31, 32] reflect the uncertainties of the experimental data used to obtain the missing input. The studies gave the following results: an uncertainty of $\mathcal{O}(100) \mathrm{MeV} / c^{2}$ for $M_{\mathrm{W}}$ and for the difference $M_{\mathrm{W}^{+}}-M_{\mathrm{W}^{-}}$, an uncertainty of $\mathcal{O}(40) \mathrm{MeV} / c^{2}$ for $\Gamma_{\mathrm{W}}$, and an uncertainty of $\mathcal{O}(0.001)$ for $\sin ^{2} \theta_{\mathrm{W}}$. Already for an integrated luminosity as small as $1 \mathrm{fb}^{-1}$ the errors that result from the uncertainties of today's missing input, are larger than statistical and systematic errors stemming from the LHC data.

The conclusion is that the current precision [1] of the above electroweak parameters cannot be improved at the LHC unless the precision of the missing input is significantly improved. This conclusion is in conflict with the prognoses made by the LHC experiments. The conflict is particularly apparent for the $\mathrm{W}$ mass where the measurement precision is found to be $5-10$ times worse than estimates made by the LHC Collaborations [4-7].

In order to ascertain the origin of these discrepancies the analysis was repeated using the LHC Collaborations' method of studying the impact of the uncertainties of $k_{\mathrm{T}}$ integrated PDFs on the electroweak observables. With the same range of uncertainty of the parameters of the $k_{\mathrm{T}}$ integrated PDFs, the results became compatible with the results of the LHC Collaborations.

The discrepancy can be traced back to two sources: a lack of considering compensating PDF changes especially in regions where such changes are hardly constrained by existing experimental data, and too rigid a restriction of the functional forms of the missing input at the initial hardness scale.

\section{Ways forward}

There is much discussion about improvements of the parton density functions from HERA experiments. The HERA programme is completed. Results from the $\mathrm{e}^{ \pm}$-proton scattering data of the $\mathrm{H} 1$ and ZEUS Collaborations have been published [33], more results from common analyses are forthcoming. Although the ultimate measurement errors are expected to be reduced by a factor of up to two, the level of uncertainty of the PDFs of $u_{v}, d_{v}$ and $s$ quarks as assumed in this paper's analysis is appropriate. The HERA data are dominated by neutral-current $\mathrm{e}^{ \pm}$-proton scattering, while the separation between quarks and antiquarks requires charged-current scattering. The scarce statistics of chargedcurrent scatterings (less than $20 \mathrm{k}$ events) render them inadequate to provide the missing input for the LHC. Moreover, the neutral-current scatterings are largely insensitive to compensating changes of the PDFs of $u_{\mathrm{v}}$ and $d_{\mathrm{v}}$ quarks.

The final results of the H1 and ZEUS Collaborations on heavy-flavour production are not yet available. However, 
again for scarce statistics, these data cannot pin down the PDFs of $\mathrm{c}$ and $\mathrm{b}$ quarks relative to those of the $\mathrm{u}$ and $\mathrm{d}$ quarks at the required level of $\sim 1 \%$.

Also, the present and the possible future experimental programme at the Jefferson Laboratory cannot improve the knowledge of the proton PDFs at the $M_{\mathrm{W}}^{2}$ and $M_{\mathrm{Z}}^{2}$ scales. This is because only a fraction of the pertinent deep-inelastic scattering data-where the higher-twists and target-mass corrections can be neglected-lends itself to QCD fits of PDFs. ${ }^{23}$ At the Jefferson Laboratory where $W_{\max }^{2}=11 \mathrm{GeV}^{2} / c^{4}$, the relevant kinematical region is beyond reach.

If, as planned, the electron beam momentum at the Jefferson Laboratory is increased to $12 \mathrm{GeV} / c$, the boundary of the useful region will be crossed but only barely so. ${ }^{24}$

\subsection{Deuteron-deuteron collisions at the LHC}

The impact of the uncertainties from missing input PDFs can be considerably reduced by operating the LHC with isoscalar beams. The natural choice is to collide deuteron beams.

The LHC luminosity is expected to scale like $\mathcal{L}_{\text {ion-ion }}=$ $\mathcal{L}_{\mathrm{pp}} / A^{2}$ where $A$ is the mass number of a light ion. Then the event rates with high $p_{\mathrm{T}}$-signatures will be comparable for the proton and for the light-ion collisions. The experimental environment at the LHC that is characterized by multiple proton-proton collisions within the same bunch-crossing, is not rendered more difficult by parasitic collisions of spectator nucleons in light-ion collisions.

The deuteron beams restore isospin symmetry for the quarks of the 1 st family. The four independent $k_{\mathrm{T}}$-integrated PDFs $u(x), d(x), \bar{u}(x)$ and $\bar{d}(x)$ are reduced to two: $u(x)+$ $d(x)$ and $\bar{u}(x)+\bar{d}(x)$. Equality of $\mathrm{W}^{+}$and $\mathrm{W}^{-}$production is restored and the spin-density matrices of $\mathrm{W}$ and $\mathrm{Z}$ produced by quarks of the 1 st family are nearly the same. ${ }^{25}$ If the contributions from quarks of the 2nd and 3rd family could be neglected, the isospin symmetry of deuterons at the LHC would play the same rôle as the matter-antimatter symmetry at the Tevatron.

The isospin symmetry of the 1st quark family reduces the number of needed two-dimensional PDFs from ten to eight. With the assumption that the PDFs of the s, c and the $b$ quark flavours are the same for quarks and antiquarks, the number of needed PDFs is further reduced from eight to

\footnotetext{
${ }^{23}$ For example, in the MSTW set of QCD fits, only data are used that satisfy the condition $W^{2}>15 \mathrm{GeV}^{2} / c^{4}$ on the squared hadronic mass [13].

${ }^{24}$ The useful data would have inelasticity $y>0.75$ where resonant photo-production processes are dominant and where QED radiative corrections are large.

${ }^{25}$ It is assumed that the $Q^{2}$-evolution from the $M_{\mathrm{W}}^{2}$ to the $M_{\mathrm{Z}}^{2}$ scale is handled as proposed in Sect. 3.4.
}

five. Given the four constraints from the measured $\mathrm{W}^{+}$and $\mathrm{W}^{-}$and $\mathrm{Z}$ cross-sections in dd collisions, there is only one two-dimensional PDF left unconstrained.

With a view to solving this problem, the sensitivity of the most sensitive electroweak parameter, the $\mathrm{W}$ mass, to the uncertainty in the $b$ quark density function has been investigated by analyzing the $\mathrm{Z}$ cross-sections not in the full pseudorapidity range $\left|\eta_{1}\right|<2.5$, but in the restricted region $2<\left|\eta_{1}\right|<2.5$. Since the contribution of $b \bar{b}$ annihilations is reduced in this kinematical region, the sensitivity of the $\mathrm{Z}$ cross-section to the $\mathrm{b}$ quark PDFs is reduced, too. Varying the b-quark PDF by $40 \%$ from its central value, the $\mathrm{W}$ mass changed by $5 \mathrm{MeV} / \mathrm{c}^{2}$, comparable with the statistical error of the pseudo-data sample. Since this is perfectly acceptable, one might conclude that taking data with deuterons in the LHC would provide the wanted precision of electroweak parameters.

However, caveats remain.

A limitation arises from the statistical error of the $\mathcal{A}_{\mathrm{W}}$ observable that measures in dd collisions directly the $s(x)-$ $c(x)$ distribution $[9,30]$. For the smallness of the Cabibbo angle, reducing the statistical error to the level sufficient to determine $M_{\mathrm{W}}$ with a precision of $10 \mathrm{MeV} / c^{2}$ requires a substantial integrated luminosity of dd collisions: $25 \mathrm{fb}^{-1}$.

The PDFs of the proton and of the neutron bound in deuterons are different with respect to the PFDs of free nucleons. The nuclear binding effects, off-shellness, and shadowing effects, could however be absorbed in a consistent way into the $\mathrm{W}$ and $\mathrm{Z}$ observables proposed in Sect. 3.2.

In summary, high-statistics data from dd collisions at the LHC would be sufficient to provide electroweak parameters with the desired precision.

\section{$4.2 \mathrm{pp}$ at the LHC, $\mathrm{p} \overline{\mathrm{p}}$ at the Tevatron, and muon-nucleon scattering combined}

The concept of solving the missing-input problem by $\mathrm{dd}$ collisions in the LHC is elegant and technically feasible, though not realistic in the near future. Therefore, an alternative is discussed: obtaining with sufficient precision from a joint analysis of Tevatron $\mathrm{p} \overline{\mathrm{p}}$ data, of data from a new muon-nucleon scattering experiment, and of LHC pp data, all needed PDFs with adequate precision.

Two intrinsic difficulties come along with this concept.

A minor difficulty is cross-normalization between pp and $p \bar{p}$ experiments with adequate precision. ${ }^{26}$ This problem can be solved by measuring the luminosity through the well-known cross-section of $1^{+} 1^{-}$pairs with a back-to-back configuration in the transverse plane, in peripheral protonproton interactions and proton-antiproton interactions, respectively [29].

\footnotetext{
${ }^{26}$ The majority of the observables are normalization-independent ratios but not all, see (12).
} 
A major difficulty is that very different $x$ domains of pertinent PDFs are populated, and that for $\mathrm{W}$ and $\mathrm{Z}$ production only the product of the $x$ values of the annihilating quarks is known, $x_{1} \cdot x_{2}$. Therefore, different $x$ intervals must be considered, ranging from 'very small $x$ ' at the LHC over 'small $x$ ' at the Tevatron until 'medium $x$ ' at the muonnucleon scattering experiment. The intervals are partially overlapping - generally, if $x_{1}$ is small, $x_{2}$ is large, and vice versa. In the following it is discussed how in a coherent way, yet with different strategies in different $x$ intervals, the information from different intervals can be linked and the missing high-precision PDFs for the analysis of LHC pp data across the full range of $x_{1}$ and $x_{2}$ be obtained.

The LHC is operated at a much higher energy than the Tevatron. Be $x_{\text {low }}^{\mathrm{LHC}}$ and $x_{\text {high }}^{\mathrm{LHC}}$ the minimal and the maximal $x$ of the quarks that produce $\mathrm{W}$ and $\mathrm{Z}$ at the LHC, and $x_{\text {low }}^{\mathrm{TEV}}$ and $x_{\text {high }}^{\mathrm{TEV}}$ their equivalents at the Tevatron. The unfolding of the PDFs will be different in the LHC-exclusive interval $\left[x_{\text {low }}^{\mathrm{LHC}}, x_{\text {low }}^{\mathrm{TEV}}\right]$, in the overlap interval $\left[x_{\text {low }}^{\mathrm{TEV}}, x_{\text {high }}^{\mathrm{LHC}}\right]$, and in the Tevatron-exclusive interval $\left[x_{\text {high }}^{\mathrm{LHC}}, x_{\text {high }}^{\mathrm{TEV}}\right]$.

It is assumed that the observables have been corrected for their $Q^{2}$ evolution and are defined at $Q^{2}=M_{\mathrm{W}}^{2}$. Likewise, the needed two-dimensional PDFs refer to $Q^{2}=M_{\mathrm{W}}^{2}$ and have no energy dependence. ${ }^{27}$

Like in Sect. 3.5, in a first step it is discussed how to determine, at fixed $x$, the ten $k_{\mathrm{T}}(x)$ densities for the $\mathrm{u}, \mathrm{d}$, $\mathrm{s}, \mathrm{c}, \mathrm{b}$ quarks and antiquarks. Then, in a second step, it is discussed how to determine the $x$ dependence of the ten $k_{\mathrm{T}^{-}}$ integrated PDFs.

The discussion proceeds in the order of increasing $x$.

- In the LHC-exclusive interval which concerns the verylow $x$ region, the analysis problem was partly discussed already in Sect. 3.5. As for the $k_{\mathrm{T}}(x)$ densities, it was concluded that the three densities $k_{\mathrm{T}}^{\mathrm{u}, \mathrm{d}, \mathrm{s}}(x), k_{\mathrm{T}}^{\mathrm{c}}(x)$, and $k_{\mathrm{T}}^{\mathrm{b}}(x)$ can be determined from the $p_{\mathrm{T}}$ dependence of the four LHC observables.

As for the $x$ dependence of the ten needed $k_{\mathrm{T}}$-integrated PDFs, the equality of the quark and antiquark densities of all five quark flavours is assumed, for $\mathrm{W}$ and $\mathrm{Z}$ are dominantly produced in the annihilation of sea quarks. ${ }^{28}$ This reduces the number of needed $k_{\mathrm{T}}$-integrated PDFs from 10 to five, still one more than can be determined from the $\eta$ distribution of the four LHC observables.

The obvious choice is to rely on the 'HERA-combination' of PDFs,

$$
4 / 9[u(x)+\bar{u}(x)+c(x)+\bar{c}(x)]
$$

\footnotetext{
${ }^{27} \mathrm{~A}$ possible small energy dependence of the $k_{\mathrm{T}}(x)$ distributions, at fixed $x$, can be corrected for.

${ }^{28}$ At $x \sim 6 \times 10^{-3}$, this assumption is violated for the $\mathrm{u}$ and $\mathrm{d}$ quarks at the $\sim 15 \%$ level which calls for an appropriate correction to be applied.
}

$$
+1 / 9[d(x)+\bar{d}(x)+s(x)+\bar{s}(x)]
$$

evolved with the DGLAP equations from the HERA- $Q^{2}$ to the $M_{\mathrm{W}}^{2}$ scale. However, this evolution algorithm that per se leads already to a $\sim 2 \%$ uncertainty at the $M_{\mathrm{W}}^{2}$ scale [33], is not undisputed for very low $x$ [34].

With the latter proviso, the problem is solved.

- In the overlap interval, the needed ten $k_{\mathrm{T}}(x)$ densities are constrained by the $p_{\mathrm{T}}$ dependence of eight observables, four from the LHC and four from the Tevatron, which are sufficiently independent of each other. Two assumptions must be made. A straightforward choice is $k_{\mathrm{T}}^{\mathrm{c}}(x)=k_{\mathrm{T}}^{\overline{\mathrm{c}}}(x)$ and $k_{\mathrm{T}}^{\mathrm{b}}(x)=k_{\mathrm{T}}^{\overline{\mathrm{b}}}(x)$.

The same assumptions can also be made for the $x$ dependence of the $k_{\mathrm{T}}$-integrated PDFs of the $\mathrm{c}$ and $\mathrm{b}$ quarks and antiquarks, ${ }^{29} c(x)=\bar{c}(x)$ and $b(x)=\bar{b}(x)$. Then from the $\eta$ dependence of the eight observables the $x$ dependences of the eight remaining $k_{\mathrm{T}}$-integrated PDFs can be determined.

The problem is solved.

- In the Tevatron-exclusive interval, the contribution of annihilations involving the bottom quark can be neglected, which reduces the needed $k_{\mathrm{T}}$ densities from ten to eight. Assuming further that $k_{\mathrm{T}}^{\mathrm{c}}(x)=k_{\mathrm{T}}^{\overline{\mathrm{c}}}(x)$, that the $k_{\mathrm{T}}$ densities of the $\mathrm{u}, \mathrm{d}$, and $\mathrm{s}$ antiquarks is the same, $k_{\mathrm{T}}^{\overline{\mathrm{u}}, \overline{\mathrm{d}}, \overline{\mathrm{s}}}(x)$, and that the $k_{\mathrm{T}}$ densities of the $\mathrm{u}$ and $\mathrm{d}$ valence quarks are the same, $k_{\mathrm{T}}^{\mathrm{u}_{\mathrm{T}}, \mathrm{d}_{\mathrm{v}}}(x)$, all four remaining $k_{\mathrm{T}}$ densities can be determined from the $p_{\mathrm{T}}$ dependence of the four observables.

As for the $x$ dependence of the eight needed $k_{\mathrm{T}}$-integrated PDFs that remain after neglecting the contributions from $\mathrm{b}$ quarks and antiquarks, it is assumed that $c(x)=\bar{c}(x)$ and $c(x)=0 .{ }^{30}$ This leaves six $k_{\mathrm{T}}$-integrated PDFs to be determined. Given only the $\eta$ dependences of four Tevatron observables, two $k_{\mathrm{T}}$-integrated PDFs remain unconstrained.

There remains a problem which can be solved only with additional data. Such data would be provided by a highprecision muon-nucleon scattering experiment.

The muon-nucleon scattering experiment would measure from the deep-inelastic scattering of $\mathcal{O}(100) \mathrm{GeV} / c$ muons on stationary hydrogen and deuterium targets the asymmetry

$\mathcal{A}_{\mathrm{DIS}}^{\mathrm{p}, \mathrm{n}}=\frac{\sigma(\mu, \mathrm{p})-\sigma(\mu, \mathrm{n})}{\sigma(\mu, \mathrm{p})+\sigma(\mu, \mathrm{n})}$

\footnotetext{
${ }^{29}$ In case that the Tevatron precision of the $\mathcal{A}_{\mathrm{Z}}$ observable is not good enough, the equality of the two-dimensional PDFs of the s quarks and antiquarks can be assumed with negligible effects on the uncertainties of the measured electroweak parameters.

${ }^{30}$ Close to the overlap interval, at $x \sim 8 \times 10^{-2}$, the latter assumption is violated at the $\sim 5 \%$ level which requires a small correction to be applied.
} 


$$
\propto u_{\mathrm{v}}-d_{\mathrm{v}}+2 \cdot(\bar{u}-\bar{d})+\text { corr. }
$$

which is-as far as the difference between $\bar{u}$ and $\bar{d}$ is concerned-complementary to what is measured by the $\mathrm{W}$ production asymmetry (see (9))

$\mathcal{A}_{\mathrm{W}} \propto u_{\mathrm{v}} \cdot \bar{d}-d_{\mathrm{v}} \cdot \bar{u}+$ corr.

It is recalled that the difference between $\bar{u}$ and $\bar{d}$ is an important ingredient for the understanding of $\mathrm{W}^{+}, \mathrm{W}^{-}$and $\mathrm{Z}$ polarizations at the $\mathrm{LHC}$.

The asymmetry $\mathcal{A}_{\mathrm{DIS}}^{\mathrm{p}, \mathrm{n}}$ has the advantage of bypassing normalization problems but provides only one constraint where two are needed. For the second constraint, the assumption $s(x)=\bar{s}(x)$ is a reasonable choice. ${ }^{31}$

With the inclusion of the muon-nucleon scattering data, the problem of missing high-precision PDFs for the analysis of LHC pp data is solved.

The present uncertainty on $\mathcal{A}_{\text {DIS }}^{\mathrm{p}, \mathrm{n}}$ from lepton-nucleon scattering experiments stems from three sources: (i) the statistical precision of (1-4)\%, (ii) uncertainties of $\sim 2 \%$ in nuclear corrections, and (iii) uncertainties of $\sim 2 \%$ in the $Q^{2}$ evolution to the $M_{\mathrm{W}}^{2}$ scale. The new muon-nucleon scattering experiment would have to reduce the statistical error by a factor of four, and improve by a comparable factor the experimental and theoretical control of uncertainties from nuclear effects in the deuteron. As for the latter, the electronnucleon scattering programme at the Jefferson Laboratory is expected to provide new insights.

A Letter of Intent [35] for such an experiment was submitted to CERN Programme Committees. Therein, the exposure of the COMPASS detector to the muon beam of the CERN-SPS was proposed.

\section{Conclusion}

The measurement of the $\mathrm{W}$ mass at the LHC with a precision of $\mathcal{O}(10) \mathrm{MeV} / c^{2}$ is per se important, and even more important if the Higgs boson will not be found. However, the prognoses by the LHC Collaborations that they can achieve this precision are much too optimistic, for the inadequate knowledge of certain proton PDFs that are not relevant in the analysis of $\mathrm{p} \overline{\mathrm{p}}$ collisions at the Tevatron but are relevant in the analysis of pp collisions at the LHC.

The missing input for the precise measurement of parameters of the Electroweak Standard Model from pp collisions at the LHC is identified. Proposals are discussed how to solve the missing-input problem. One possibility is to

\footnotetext{
${ }^{31}$ This assumption can be questioned; in this case, rather than measuring the asymmetry $\mathcal{A}_{\text {DIS }}^{\mathrm{p}, \mathrm{n}}$, absolute cross-sections $\sigma(\mu, \mathrm{p})$ and $\sigma(\mu, \mathrm{n})$ would have to measured.
}

complement the pp programme of the $\mathrm{LHC}$ with a deuterondeuteron collision programme. Another possibility is to obtain missing input from a new high-precision muon-nucleon scattering experiment, and to analyze these data coherently with LHC pp and Tevatron $\mathrm{p} \overline{\mathrm{p}}$ data. In the framework of an LHC-specific programme for the precision measurement of parameters of the Electroweak Standard Model, a precision of $10 \mathrm{MeV} / c^{2}$ of $M_{\mathrm{W}}$ can be achieved.

Unless efforts as discussed in this paper are undertaken, the precision of the $\mathrm{W}$ mass, and of other parameters of the Electroweak Standard Model, will not be improved at the LHC. Thus a chance may be missed towards understanding the mechanism that regularizes the unitarity problem of this Model.

Open Access This article is distributed under the terms of the Creative Commons Attribution Noncommercial License which permits any noncommercial use, distribution, and reproduction in any medium, provided the original author(s) and source are credited.

\section{References}

1. C. Amsler et al. (Particle Data Group), Phys. Lett. B 667, 1 (2008)

2. The Tevatron Electroweak Working Group, Updated Combination of CDF and D0 results for the Mass of the W Boson, FERMILABTM-2439-E, arXiv:0908.1374 [hep-ex]

3. The LEP Electroweak Working Group, A Combination of Preliminary Electroweak Measurements and Constraints on the Standard Model, CERN-PH-EP-2006-042, arXiv:hep-ex/0612034

4. ATLAS Detector and Physics Performance, Report ATLAS TDR 15 (CERN/LHCC 99-15)

5. N. Besson et al. (ATLAS Collaboration), Eur. Phys. J. C 57, 627 (2008). arXiv:0805.2093 [hep-ex]

6. N. Besson (for the ATLAS Collaboration), Presentation at the Int. Europhysics Conference on High Energy Physics (EPSHEP2009), Cracow (Poland), July 2009

7. V. Buge et al. (CMS Collaboration), J. Phys. G 34, 995 (2007) CERN-CMS-NOTE-2006-061

8. T. Sjostrand, S. Mrenna, P. Skands, J. High Energy Phys. 05, 26 (2006)

9. F. Fayette et al., Eur. Phys. J. C 63, 33 (2009). arXiv:0812.2571

10. J. Stirling, MSTW2008: Parton distributions for the LHC, Seminar given at CERN on 18 February, 2009

11. A.D. Martin, R.G. Roberts, W.J. Stirling, R.S. Thorne, Eur. Phys. J. C 14, 133 (2000)

12. P.M. Nadolsky et al., Phys. Rev. D 78, 013004 (2008). arXiv:0802.0007 [hep-ph]

13. A.D. Martin W.J. Stirling, R.S. Thorne, G. Watt, Eur. Phys. J. C 63, 189 (2009). arXiv:0901.0002v2 [hep-ph]

14. M. Ubiali et al. (NNPDF Collaboration), arXiv:1005.0397, and references quoted therein

15. R.D. Ball et al. (NNPDF Collaboration), arXiv:1002.4407

16. M.R. Whalley, D. Bourilkov (and R.C. Group) arXiv:hep-ph/ 0508110

17. W. Płaczek, S. Jadach, http://cern.ch/placzek/winhac

18. W. Płaczek, S. Jadach, Eur. Phys. J. C 29, 325 (2003)

19. M.W. Krasny et al., Eur. Phys. J. C 51, 607 (2007). hep-ph/0702251

20. C.M. Carloni Calame et al., Acta Phys. Pol. B 35, 1643 (2004). arXiv:hep-ph/0402235 
21. C.E. Gerber et al. (TeV4LHC-Top and Electroweak Working Group), arXiv:0705.3251

22. D. Bardin et al., Acta Phys. Pol. B 40, 75 (2009). arXiv:0806.3822

23. W. Płaczek, WINHAC - the Monte Carlo event generator for single W-boson production in hadronic collisions, arXiv:0911.0572 [hep-ph]

24. A. Siódmok, A.W. Płaczek, ZINHAC - the Monte Carlo event generator for single Z-boson production in hadronic collisions. http://th-www.if.uj.edu.pl/ZINHAC

25. D. Stump et al., J. High Energy Phys. 10, 46 (2003). arXiv:hep-ph/0303013

26. A.D. Martin, W.J. Stirling, R.G. Roberts, Phys. Rev. D 50, 6734 (1994). arXiv:hep-ph/9406315

27. T. Aaltonen et al. (CDF Collaboration), Phys. Rev. D 77, 112001 (2008)

28. V.M. Abazov et al. (D0 Collaboration), Phys. Rev. Lett. 103, 141801 (2009)
29. M.W. Krasny, J. Chwastowski, K. Slowikowski, Nucl. Instrum. Methods Phys. Res. A 584, 42 (2008). arXiv:hep-ex/0610052

30. F. Fayette, Strategies for precision measurements of the charge asymmetry of the $\mathrm{W}$ boson mass at the LHC within the ATLAS experiment. arXiv:0906.4260 [hep-ex], PhD thesis

31. K. Rejzner, F. Fayette, M.W. Krasny, W. Placzek, A. Siodmok, PoS HCP2009, 095 (2009)

32. M.W. Krasny et al., Measurement of $\sin ^{2} \theta_{\mathrm{W}}$ at the LHC, paper in preparation

33. H1 and ZEUS Collaborations, Combined measurement and QCD analysis of the inclusive $\mathrm{e}^{ \pm} \mathrm{p}$ scattering cross sections at HERA. Preprint DESY 09-158. arXiv:0911.0884 [hep-ex]

34. K.J. Golec-Biernat, M.W. Krasny, S. Riess, Phys. Lett. B 337, 367 (1994)

35. F. Dydak, M.W. Krasny, R. Voss, Document CERN-SPSC-2009028/CERN-LHCC-2009-014 